\title{
Situation of COVID-19 in Brazil in August 2020: An Analysis via Growth Models as Implemented in the ModInterv System for Monitoring the Pandemic
}

\author{
Giovani L. Vasconcelos ${ }^{1}$ (D) - Gerson C. Duarte-Filho ${ }^{2} \cdot$ Arthur A. Brum $^{3} \cdot$ Raydonal Ospina $^{4}$. \\ Francisco A. G. Almeida ${ }^{2}$. Antônio M. S. Macêdo ${ }^{3}$
}

Received: 17 February 2021 / Revised: 4 October 2021 / Accepted: 13 October 2021 / Published online: 3 January 2022

(c) Brazilian Society for Automatics-SBA 2021

\begin{abstract}
In this work we introduce a novel methodology to classify the dynamical stages of an epidemic, based on the different acceleration regimes of the corresponding growth curve. Our classification scheme is implemented by fitting the empirical data with a general class of mathematical growth models, from which we compute not only the growth acceleration but also its jerk and jounce (i.e., the first and second derivatives of the acceleration, respectively), thus allowing for a finer distinction of the epidemic stages. Using this methodology, we analyze the cumulative curves of deaths attributed to COVID-19 in the 26 Brazilian States and the Federal District, up until August 21, 2020. The online application ModInterv COVID-19, which automatically implements the classification scheme and which can be accessed via an internet browser or a mobile app, was used to investigate the epidemic stages in each of the Brazilian federal units. The analysis revealed that almost all states in the Northern and Northeastern regions were already in the saturation phase, meaning that the epidemic was relatively under control, whereas in all Southern states and in most states in the Midwest the epidemic was still accelerating or showed only a slight deceleration. The Southeastern region presented a great diversity of epidemic stages, with each state being found at a different stage, ranging from acceleration to saturation. It is argued that understanding this heterogeneous geographical distribution of the epidemic is relevant for public health authorities, as it may help in devising more effective strategies against the COVID-19 pandemic in a continental country like Brazil.
\end{abstract}

Keywords COVID-19 · Epidemiological models · Growth models · Generalized logistic model $\cdot$ Public health policies

\section{Introduction}

This article is dedicated to the memory of Firmino Soares-Filho, former Mayor of Teresina, Brazil, and a strong supporter of our research on epidemic dynamics.

Giovani L. Vasconcelos

giovani.vasconcelos@ufpr.br

Gerson C. Duarte-Filho

gcdf@ufs.br

Arthur A. Brum

arthurbrum@df.ufpe.br

Raydonal Ospina

raydonal@de.ufpe.br

Francisco A. G. Almeida

falmeida@ufs.br

Antônio M. S. Macêdo

antonio.smacedo@ufpe.br
In Brazil, after more than five months since the first case of infection by the new coronavirus (SARS-CoV-2) and the first death from the disease (COVID-19) caused by the virus, the cumulative numbers of cases and deaths attributed to the disease showed no sign of stagnation. At the time of the writing of the first version of this paper, namely, in late August 2020, Brazil had more than 3.5 million confirmed cases and more

1 Departamento de Física, Universidade Federal do Paraná, Curitiba, PR 81531-990, Brazil

2 Departamento de Física, Universidade Federal de Sergipe, São Cristóvão, SE 49100-000, Brazil

3 Departamento de Física, Universidade Federal de Pernambuco, Recife, PE 50670-901, Brazil

4 Departamento de Estatística, CASTLab, Universidade Federal de Pernambuco, Recife, PE 50740-540, Brazil 
than 110,000 deaths due to the disease (Johns Hopkins University 2021; Worldometer 2020). Looking at the situation of the epidemic, state by state, it was possible to observe that some of them seemed to be in a phase where the cumulative numbers of cases as well as of deaths still presented an accelerated growth, while other states seemed to have reached a stage where the numbers were growing more slowly or were already in a stage of saturation. In other words, there was a strong geographical heterogeneity of the epidemic in Brazil. In this complex context, it is therefore important to know the evolution of the COVID-19 epidemic in each of the federal units, since local governors had the legal autonomy to decide on the measures to be adopted to counter the propagation of the virus within their jurisdictions. Understanding the differences and similarities of the epidemic trajectories in the several regions of a continental country like Brazil may also help government and public health authorities to devise more effective strategies against the COVID-19 pandemic.

A common way to track the evolution of an epidemic in a given locality is through the effective reproduction number $R_{t}$, where we recall that $R_{t}>1\left(R_{t}<1\right)$ implies that the epidemic is accelerating (decelerating), whereas $R_{t}=1$ corresponds to linear growth. Although $R_{t}$ is widely used, the determination and interpretation of this quantity can sometimes be problematic (Anderson et al. 2020; Adam 2020). For instance, one problem with determining $R_{t}$ is that it depends on epidemiological quantities that are not readily available from the epidemic data, such as the average time between successive infection events. Another important caveat to bear in mind about $R_{t}$ is that it can reach identical values at different stages of an epidemic outbreak, which renders this parameter (by itself) insufficient to fully monitor the epidemic evolution. To be more precise, since $R_{t}$ is essentially a (dimensionless) measure of the epidemic acceleration, it will assume repeated values around any maximum or minimum of the acceleration curve. In other words, $R_{t}$ cannot distinguish between regimes of increasing or decreasing acceleration/deceleration, as it depends only on the acceleration itself. Other ways of monitoring the evolution of an epidemic in terms of acceleration parameters have been recently discussed in the literature (Utsunomiya et al. 2020; Baunez et al. 2021; Rebhan 2021). These alternative metrics have the advantage that they can be obtained directly from the epidemic curves, but they also share the limitation with $R_{t}$ that they are based either solely on the growth acceleration or, at most, on its first derivative (Utsunomiya et al. 2020). Hence, they can distinguish only up to regimes of growing or decreasing acceleration.

In the present paper, we introduce a novel methodology to classify the stages of an epidemic in a given place. Our classification scheme is based not only on the value of the growth acceleration but also on its first and second derivatives, known as the jerk (or jolt) and the jounce (or snap), respec- tively. This allows us to distinguish, for example, among different regimes of increasing acceleration, depending on whether the jerk is increasing or decreasing (i.e., whether the jounce is positive or negative). Similar classification is also possible for regimes of decreasing deceleration but with different signs of the jounce.

To implement this classification scheme, we employ a general class of mathematical growth models to fit the cumulative epidemic curves. The theoretical curve then allows us to compute analytically the characteristic points that separates the different stages of the evolution, namely the point of zero acceleration (inflection point) as well as the points of zero jerk and zero jounce. By comparing the location of the last data point of the empirical curve in relation to these characteristic points, one can then ascertain the current stage of evolution of the epidemic curve. Furthermore, by analyzing how close the last data point is of the next characteristic point one can also make some prediction as to when the epidemic is likely to progress to the subsequent dynamical regime.

Our methodology thus allows for a finer classification scheme of the different growth regimes of an epidemic and, by doing so, can provide useful information to health authorities not only in regard to the current stage of the epidemic in the locality of interest but also to its likely evolution in the near future. This can help guiding the authorities in their decision making process regarding, say, the implementation or relaxation of non-pharmacological interventions. Furthermore, from a numerical viewpoint, our method requires only a fit to the cumulative epidemic curve with a general class of mathematical growth models, from which the points separating the different dynamical stages can be computed analytically, so that the classification of a given epidemic curve can be performed automatically on the computer (i.e., without human assistance), thus making the method easily accessible to any interested person, once the method is algorithmically implemented.

The classification scheme discussed here is, however, limited to epidemic curves that display a sigmoidal shape, meaning that second-wave effects are not contemplated. In principle, it would be possible to extend our classification method to multiple-wave epidemics. But in such cases one needs to consider growth models with time-dependent parameters (Vasconcelos et al. 2021a), so that the characteristic points can no longer be obtained analytically, which would render the method more numerically demanding. Classification of epidemic curves with second or more waves will therefore be left for future investigation.

Our classification scheme has been implemented in the application ModInterv COVID-19 (Brum et al. 2020), developed by our Research Network on Modeling the COVID-19 Epidemic and Non-Pharmacological Interventions MODINTERV-COVID19 (http://fisica.ufpr.br/redecovid19). The application is available for general use via the internet 
(http://fisica.ufpr.br/modinterv) or via an Android mobile app available free at the Play Store (https://play.google.com/ store/apps/details?id=com.tanxe.COVID_19). In the ModInterv application, it is possible to monitor the cumulative and daily curves of cases and deaths of COVID-19 for all countries worldwide as well as for the states and cities in Brazil and the United States. Once a location is chosen, the algorithm employs a general class of mathematical growth models to fit the selected cumulative curve of cases or deaths. From the predictions of the model that best fits the selected empirical curve, and applying the classification scheme briefly described above (see below for more details), the Modlnterv app then informs the current stage of the epidemic in the chosen location.

In this paper, we apply our epidemic classification method, as implemented in the Modlnterv app, to obtain an overview of the evolution of the COVID-19 epidemic in the 26 states of Brazil and the Federal District, up to August 21, 2020. From the predictions of the app, it is then possible to determine the evolution stages of the epidemic in each of the Brazilian federal units. Here we have chosen to analyze the situation in Brazil only up to mid-August, 2020, because at that time all five stages of the COVID-19 epidemic defined in the ModInterv were still represented in Brazil. In other words, this date corresponds to a period of maximum diversity in the evolution patterns of the first wave of COVID-19 in Brazil.

Hence, it is an important epidemiological moment that deserves to be described and understood in more detail. Indeed, as we will see later, by comparing the different stages of COVID-19 pandemic in the different states and regions of Brazil, one can identify several patterns as to how the epidemic evolved geographically in the country (up to the data chosen). Although here we have restricted ourselves to analyzing the situation of COVID-19 in the Brazilian states, the app Modlnterv can be used to perform similar analysis in other places of interest. However, when analyzing an epidemic curve with more than one wave, it is necessary to confine the analysis to the data prior to the onset of the second wave, as our classification scheme is, as of now, limited to the first wave of an epidemic.

\section{Data}

In the present work, we analyze the cumulative curves of deaths attributed to COVID-19 for the 26 states of Brazil and the Federal District, with data updated until the date of August 21, 2020. The free data used in our analysis are automatically "downloaded" from the website COVID19br.wcota.me, which in turn compiles and updates the data from the bulletins published by the respective State Health Departments (Cota 2020). The mortality data are normally tabulated according to the day on which each death occurred. Thus, an epidemic curve for a given State represents the accumulated number of deaths, $C(t)$, attributed to COVID-19 that occurred in that State up to the time $t$, counted in days since the first death.

It is inevitable that there may be some level of uncertainty in the data collection, such as under-reporting or delay in confirming deaths, but in general the data on deaths are considered more reliable than the data for infection cases, which are admittedly much more affected by the under-reporting problem, since many infections go undetected. For this reason, we have decided to restrict our analysis to the COVID-19 death curves. As already mentioned, here we consider the fatality curves of each Brazilian federal unit up to August 21, 2020. This upper limit date was chosen for our study because it corresponds to a period in which all five stages of the COVID-19 epidemic were still represented in Brazil.

\section{Methodology}

\subsection{Epidemic Curves and Stages}

A typical epidemic curve (Keeling and Rohani 2011), representing the cumulative number, $C(t)$, of deaths as a function of the time $t$, counted in days from the day of the first death, is illustrated in Fig. 1a. As indicated in this figure, the epidemic curve has two main phases, namely: (i) a phase of accelerated growth, that is, with positive acceleration; and (ii) a phase with decelerated growth, where the acceleration is negative. In the picture described in Fig. 1, we represent an idealized situation where stochastic effects are neglected. In real epidemics, the daily numbers of cases and deaths display a considerable degree of fluctuations. The type of smooth curve exemplified, say, in Fig. 1b, thus describes a sort of mean behavior of the real (stochastic) dynamics.

These two phases are separated by the inflection point, $t_{c}$, of the cumulative curve, which corresponds to the point where the acceleration is zero. In Fig. 1b, we show the 'velocity' curve, $\dot{C}(t)$, where dot denotes time derivative, which corresponds to the daily number of deaths. As illustrated in this figure, the inflection point, $t_{c}$, of the cumulative curve corresponds to the "peak" of the daily curve, which separates the phases of increasing and decreasing growth velocity, respectively.

From a more practical viewpoint, a finer classification scheme of the different growth regimes can be given as follows: (i) the early growth regime, where the acceleration is always increasing; (ii) the intermediate growth regime, which is characterized by a nearly-linear profile around the inflection point; and (iii) the late growth regime, for which the deceleration (i.e., the absolute value of the negative acceleration) is always decreasing. 
Note that in the late growth regime, the curve bends away from the linear regime and thus starts its approach toward the final plateau, represented by the horizontal dashed line in Fig. 1a. Moreover, each of these three growth regimes can be further divided into two stages, on the basis on the respective trends of the acceleration curve in each regime, as described below.

In Fig. 1c, we show the acceleration curve corresponding to the cumulative curve of Fig. 1a. As shown in Fig. 1c, the acceleration curve has five important characteristic points, namely: (i) the inflection point, $t_{1}$, in the region of positive acceleration; (ii) the point, $t_{2}$, of maximum acceleration; (iii) the point, $t_{3}$, of zero acceleration, which corresponds to the inflection point of the cumulative curve, i.e., $t_{3}=t_{c}$, where $\ddot{C}\left(t_{c}\right)=0$; (iv) the point, $t_{4}$, of minimum acceleration, which corresponds to the point of maximum deceleration; and (v) the inflection point, $t_{5}$, in the region of negative acceleration.

Recalling that the rate of change of the acceleration is known as the "jerk" (or "jolt"), we thus have that $t_{2}$ and $t_{4}$ are the points of zero jerk, meaning that $\dddot{C}\left(t_{2}\right)=\dddot{C}\left(t_{4}\right)=0$; while $t_{1}$ and $t_{5}$ are the points of maximum jerk or zero jounce, i.e., $\dddot{C}\left(t_{1}\right)=\dddot{C}\left(t_{5}\right)=0$, where we recall that the derivative of the jerk is known as jounce (or snap).

In view of the existence of the five characteristic points, $t_{i}, i=1, \ldots, 5$, discussed above, we can accordingly define six dynamical stages, as follows:

1. Increasing acceleration and increasing jerk (dark red stage): $t<t_{1}$.

2. Increasing acceleration and decreasing jerk (light red stage): $t_{1}<t<t_{2}$.

3. Decreasing acceleration (orange stage): $t_{2}<t<t_{3}$.

4. Increasing deceleration (yellow stage): $t_{3}<t<t_{4}$.

5. Decreasing deceleration and increasing jerk (green stage): $t_{4}<t<t_{5}$.

6. Decreasing deceleration and decreasing jerk (blue stage): $t>t_{5}$.

These six dynamical stages are indicated by the respective colors in the three curves shown in Fig. 1. Note that the early growth regime has two dynamical stages, indicated by dark and light red in Fig. 1a, respectively. In this regime, the acceleration is always increasing, see Fig. 1c, with the two stages being differentiated only as to whether the jerk is increasing (dark red) or decreasing (light red).

From a practical viewpoint, however, the distinction between these two early growth stages is not very relevant, since the first stage is relatively short.

Therefore, these two initial dynamical stages will be treated in our classification scheme (see below) as only one epidemic stage, namely that of increasing acceleration.

The intermediate growth regime comprises two epidemic stages, namely, the stage of decreasing acceleration (orange),

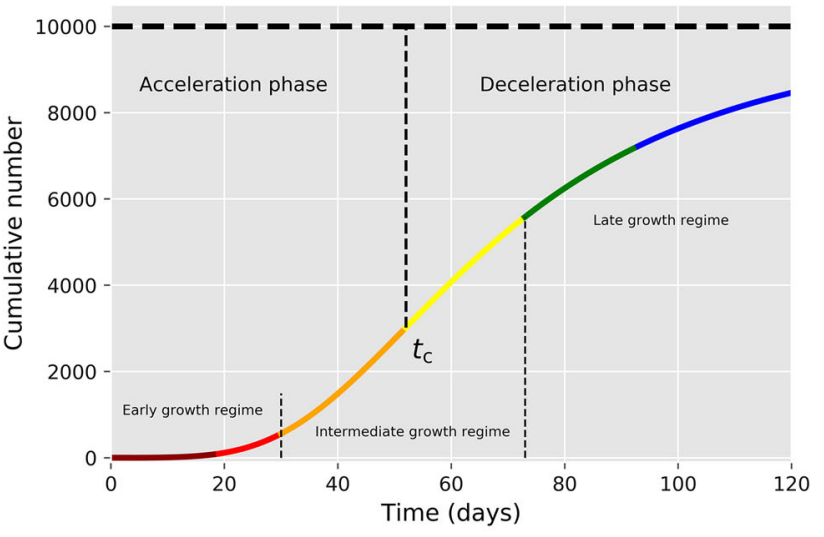

(a)

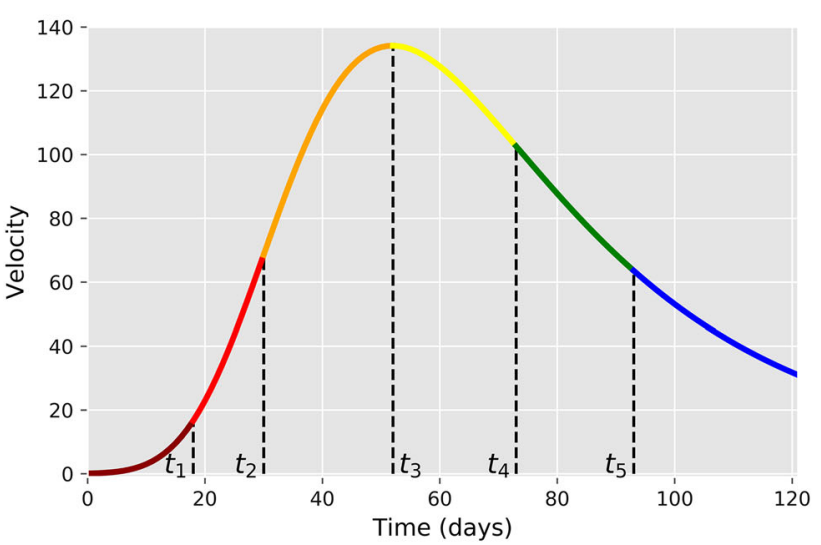

(b)

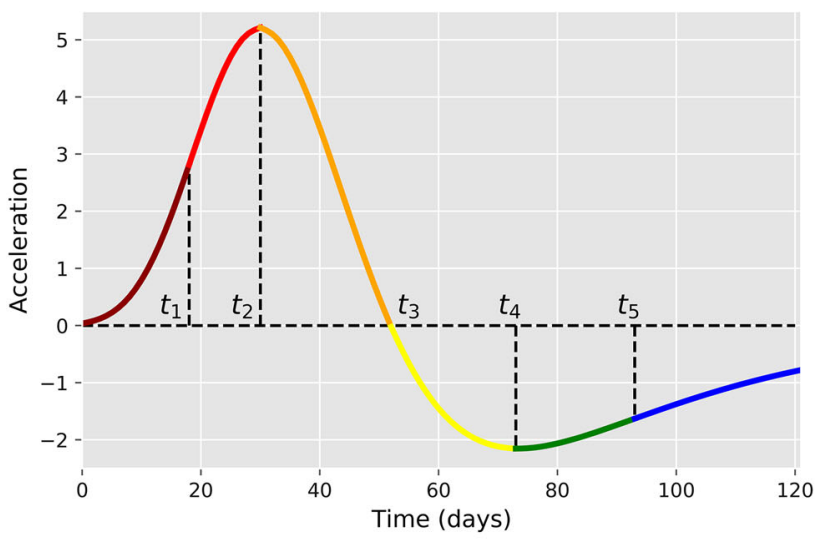

(c)

Fig. 1 a Schematic of an epidemic curve for the cumulative number of deaths, with an indication of its two main phases, namely the acceleration and deceleration phases, respectively. The point $t_{c}$ indicates the inflection point of the cumulative curve, where the acceleration is zero, which separates the two phases. b Velocity and $\mathbf{c}$ acceleration curves, corresponding to the first and second derivatives, respectively, of the cumulative curve shown in (a). The different colors in the three curves indicate the respective epidemic stages, according to the corresponding acceleration regimes 
followed by the stage of increasing deceleration (yellow), as seen in Fig. 1. Note that during the intermediate regime the growth profile is approximately linear, since the two corresponding stages are close to the inflection point, $t_{c}$, at which the acceleration is zero, thus leading to a nearly linear growth around it.

Finally, the late growth regime corresponds to the regime of decreasing deceleration, which entails two dynamical stages, depending on whether the jerk is increasing (green) or decreasing (blue). As this regime begins, the cumulative curve starts to bend more strongly, thus moving away from the quasi-linear behavior of the intermediate regime. In this sense, the first stage (green) of the late growth regime marks the transition toward the saturation of the epidemic curve, and so we shall refer to this stage as transition to saturation.

Similarly, the last stage (blue) corresponds to the saturation itself, where the first three derivatives of $C(t)$ are all decreasing, and so it may be said that the epidemic is relatively under control once it enters this final stage. (If, however, control measures are relaxed prematurely, there may be a recrudescence of infections and the death curve may accelerate again, causing the so-called second wave of infections. These effects, however, will not be considered in this analysis).

We emphasize that the discussion of the main dynamical features of an epidemic curve presented above is not model dependent. In other words, the dynamical stages shown in Fig. 1 are expected to be observed (at least in an approximated sense) for most empirical epidemic curves, so long as one restricts the data to the first wave. In the case of COVID-19 in particular, there is ample empirical evidence (Vasconcelos et al. 2020, 2021b) that the first wave of the epidemic in many countries had a sigmoidal-like shape, as illustrated in Fig. 1a. A mathematical model is, however, required to precisely identify the main acceleration regimes of a given epidemic curve, as one needs to compute the characteristic points $t_{i}$ defined above. Knowledge of the characteristic points associated with the epidemic curve of a given location then allows one to identify the current stage of the epidemic in that location, as described next.

Given a real epidemic curve at a given current time $t_{f}$, it is important to determine in which dynamical stage the epidemic is at the chosen location at that time. To do so, it is first necessary to have a mathematical model that provides a theoretical curve describing the data, from which one can calculate the characteristic points $t_{i}, i=1, \ldots, 5$, discussed above. Once those points have been obtained, one can then compare the position of the time $t_{f}$ with respect to the characteristic points $t_{i}$ obtained from the mathematical model. The stage of the epidemic can thus be determined according to the following classification scheme (and respective color code):
1. Stage of increasing acceleration (red): $t_{f}<t_{2}$.

2. Stage of decreasing acceleration (orange): $t_{2}<t_{f}<t_{3}$.

3. Stage of increasing deceleration (yellow): $t_{3}<t_{f}<t_{4}$.

4. Transition to saturation (green): $t_{4}<t_{f}<t_{5}$.

5. Saturation stage (blue): $t_{f}>t_{5}$.

The Modlnterv system, to be discussed below, implements four mathematical growth models, with different levels of complexity, from which the dynamical stage of any given epidemic curves (with no second-wave effects) can be determined.

\subsection{Mathematical Models}

The most general model, of which the others are particular cases, is the beta logistic model (BLM), described by the following ordinary differential equation (Tsoularis and Wallace 2002; Vasconcelos et al. 2021b):

$$
\frac{\mathrm{d} C}{\mathrm{~d} t}=r[C(t)]^{q}\left[1-\left(\frac{C(t)}{K}\right)^{\alpha}\right]^{p}
$$

where $r$ is the growth rate in the initial stage, $K$ is the final size of the epidemic, $q$ controls the initial growth regime and allows to interpolate from linear growth $(q=0)$ to subexponential growth $(q<1)$ to purely exponential growth $(q=1)$, The exponent $p$ controls the approach rate to the plateau, with $p>1$ implying a polynomial rate, while $p=1$ produces an exponentially fast approach, and exponent $\alpha$ is the socalled asymmetry parameter. BLM has as particular cases of interest the following models: the generalized Richards model (GRM), when $p=1$; the Richards model (RM), for $q=p=1$; and the $q$-exponential model, in case $p=0$. More details about the above models can be found in the references (Tsoularis and Wallace 2002; Vasconcelos et al. 2021b, 2020).

BLM also retrieves the standard logistic model, in which case $q=p=\alpha=1$, and therefore Eq. (1) is also known as the generalized logistic equation (Tsoularis and Wallace 2002). It is worth remembering, however, that the logistic model describes a symmetric growth curve in relation to the inflection point. But since this symmetry is rarely observed in the empirical curves of COVID-19, this particular case is not implemented separately in Modlnterv. Note, however, that in the case of the Richards model, where $q=p=1$, the exponent $\alpha$ is free to vary, so that a symmetric curve can in principle be obtained if the numeric adjustment returns the value $\alpha=1$.

The BLM admits an analytic solution (Vasconcelos et al. 2021b) in the following implicit form:

$t=f(C)-f\left(C_{0}\right)$, 
where

$f(C)=\frac{C^{1-q}}{r(1-q)}{ }_{2} F_{1}\left(p, \frac{1-q}{\alpha} ; 1+\frac{1-q}{\alpha} ;\left(\frac{C}{K}\right)^{\alpha}\right)$,

with ${ }_{2} F_{1}(a, b ; c ; x)$ being the Gauss hypergeometric function.

The inflection point of the BLM curve, $C(t)$, is located at the time $t_{3}=f\left(K x_{3}\right)$, where

$x_{3}=\left[\frac{q}{q+\alpha p}\right]^{1 / \alpha}$.

Two other important characteristic points of the curve $C(t)$ are the points of maximum and minimum acceleration, denoted by $t_{2}$ and $t_{4}$, respectively; see Fig. $1 \mathrm{~b}$. Note that since the $\ddot{C}\left(t_{4}\right)<0$, the point $t_{4}$ corresponds in fact to the point of maximum deceleration (i.e., maximum value of $|\ddot{C}(t)|$ within the region of negative acceleration). Recalling that the rate of acceleration is known as the jerk (or jolt), we thus have that the points $t_{2}$ and $t_{4}$ are the points of zero jerk, which can be obtained from the condition $\dddot{C}(t)=0$. After some tedious algebra, one finds that $t_{2,4}=f\left(K x_{2,4}\right)$, where

$x_{2,4}=\left[\frac{\alpha^{2} p+\theta-\alpha(p-4 p q \pm \sqrt{\Delta})}{4 \alpha^{2} p^{2}+\theta+2 \alpha p(-1+4 q)}\right]^{1 / \alpha}$,

where $\theta$ and $\Delta$ are given by $\theta=2 q(-1+2 q)$ and

$\Delta=4 p q(-1+2 q)+p^{2}\left(1-2 \alpha+\alpha^{2}+8 \alpha q\right)$,

respectively. One can also compute the points $t_{1}$ and $t_{5}$ of maximum jerk, i.e., $\dddot{C}(t)=0$. But in this case the expressions are prohibitively long and will not be given here.

Setting $p=1$ in Eqs. (3)-(5) yields the exact solution for the GRM, together with the corresponding expressions for its characteristic points $t_{2}, t_{3}$, and $t_{4}$. One important distinction between the general BLM (i.e., with $p>1$ ) and the GRM $(p=1)$ is that the former predicts a polynomial approach to the plateau (Vasconcelos et al. 2021b):

$K-C(t) \propto \frac{1}{t^{v}}, \quad$ for $t \gg t_{c}$

where $v=1 /(p-1)$. In contradistinction, the GRM describes an exponentially fast approach to the plateau:

$K-C(t) \propto \exp \left(-\frac{r \alpha t}{K^{1-q}}\right)$

Furthermore, for $p=q=1$, the BLM reduces to the RM, as already mentioned, in which case one obtains an explicit solution in the following form (Vasconcelos et al. 2020):

$C(t)=\frac{K}{\left\{1+\alpha \exp \left[-\alpha r\left(t-t_{c}\right)\right]\right\}^{1 / \alpha}}$,

where the inflection point $t_{c}$ can be obtained in terms of the initial condition $C_{0}$ via the relation: $C_{0}=K /[1+\alpha \exp$ $\left.\left(\alpha r t_{c}\right)\right]^{1 / \alpha}$. For the RM, the points, $t_{2}$ and $t_{4}$, of maximum acceleration and maximum deceleration, can be obtained explicitly by solving $\dddot{C}(t)=0$, which yields

$t_{2,4}=t_{c}-\frac{1}{r} \ln \left(\frac{\alpha+3 \pm \sqrt{\alpha^{2}+6 \alpha+5}}{2}\right)$.

Again, the expressions for the points $t_{1}$ and $t_{5}$ of maximum jerk are rather long and will not be given here.

When the outbreak in a given place is in its early stage, i.e., when the epidemic curve is still in a regime of increasing acceleration, see Fig. 1b, none of the previous models (RM, GRM and BLM) are suitable, because the parameters estimated from these three models (if they converge at all) are not reliable. More specifically, as the empirical curve does not exhibit yet an inflection point, estimating the parameter $\alpha$ becomes unreliable. In this case, it is preferable to use the so-called generalized growth model (Chowell 2017; Vasconcelos et al. 2020), which is defined by setting $C \ll K$ in (1), which results in the following ODE:

$\frac{\mathrm{d} C}{\mathrm{~d} t}=r[C(t)]^{q}$

The solution of (10) is

$C(t)=\left[C_{0}^{1-q}+(1-q) r t\right]^{1 /(1-q)}=C_{0} \mathrm{e}_{q}\left(\frac{r t}{C_{0}^{1-q}}\right)$

where the function $\mathrm{e}_{q}(x)=[1+(1-q) x]^{1 /(1-q)}$ is known in the physics literature as the $q$-exponential function (Picoli et al. 2009). For this reason, we shall also refer to model (10) as the $q$-exponential model. In particular, note that according to (11) the growth of the epidemic curve in this regime is approximately polynomial (for $0 \leq q<1$ ) as $t$ increases:

$C(t) \propto t^{\mu}$,

where $\mu=1 /(1-q)$, whereas only for $q=1$ does one obtain an exponential growth. Polynomial early growth has been identified in the COVID-19 data for several countries (Wu et al. 2020; Manchein et al. 2020; Vasconcelos et al. 2020). 
As indicated above, in the early growth regime (say, when a possible inflection point is not yet discernible in the empirical cumulative curve), it is more convenient to describe the data with the $q$-exponential model, as it contemplates a range of accelerated growth, from sub-exponential $(q<1)$ to fully exponential $(q=1)$. The $q$-exponential model also has the advantage that it readily provides a formula for the doubling time, $T_{d}(t)$, which corresponds to the time necessary for the number of deaths (or case) to double from its current value at time $t$.

To determine $T_{d}$, we first equate $C\left(t+T_{d}\right)=2 C(t)$, where $C(t)$ is as given in (10). After a trivial algebraic manipulation, one obtains

$T_{d}(t)=\left(2^{1-q}-1\right)\left[t+\frac{C_{0}^{1-q}}{(1-q) r}\right]$.

One then sees that the doubling time in the $q$-exponential model grows linearly in time for $q<1$, whereas it remains constant, i.e., $T_{d}=(\ln 2) / r$, for the purely exponential growth $(q=1)$, as is well known. The fact that $T_{d}$ increases as a linear function of time for $q<1$ is another manifestation of the slower than exponential growth in such cases. The $q$-exponential thus allows us to make relevant short-term predictions when the epidemic is in its early growth phase (Vasconcelos et al. 2020).

Epidemic dynamics can be described by many mathematical models, such as compartmental models of the SIR type (Kermack and McKendrick 1927; Keeling and Rohani 2011), phenomenological growth models (Bürger et al. 2019; Vasconcelos et al. 2021b), agent-based models (Shamil et al. 2021; Almagor and Picascia 2020), and purely statistical models (El-Morshedy et al. 2021; Read et al. 2021). Growth models have the mathematical advantage that they often admit analytical solutions. Furthermore, they seek to capture the basic aspects of the epidemic dynamics, without having to describe epidemiological mechanisms that may be difficult to identify (Bürger et al. 2019; Vasconcelos et al. 2020). It is worth emphasizing, however, that there is an intrinsic connection between growth models and mechanistic models of the SIR type. For instance, it is possible to construct a map between the Richards growth model and a modified SIR model (Wang et al. 2012; Macêdo et al. 2021).

There is also a connection between the BLM and a generalized SIRD model (Vasconcelos et al. 2021a). Through such maps, the growth model parameters can be put in correspondence with the parameters of the compartmental models. For example, in the map between the RM and the SIR model (Wang et al. 2012), one obtains a one-to-one connection between the parameters $(r, \alpha)$ of the RM and the SIR parameters $(\beta, \gamma)$. This relation is, however, highly nonlinear, so it is not easy to identify specific epidemiological mechanisms that separately govern the growth rate $r$ and the asymmetry parameter $\alpha$. Nonetheless, growth models are bonafide epidemiological models and, when properly applied and interpreted, can provide useful insights into the spread of novel infectious diseases (Vasconcelos et al. 2020, 2021b).

The application ModInterv implements in Python the four growth models discussed above, namely, BLM, GRM, RM, and $q$-exponential. More specifically, for a given epidemic curve, the system initially tries to adjust the empirical data with the BLM. In general, however, the convergence of this model only happens for curves that already are in a saturation phase, which allows estimating the exponent $p$ that controls the approach to the plateau. If there is no convergence of BLM, the Modlnterv next tests the GRM. As already mentioned, the GRM assumes an exponential behavior in the saturation phase, but still requires the numerical adjustment of two exponents: the exponent $q$, which controls the subexponential growth at the beginning, and the exponent of asymmetry $\alpha$. For this reason, the GRM only produces a good adjustment when the curve is already in the deceleration phase.

In cases where the GRM does not converge satisfactorily, then the we apply the RM. Since the RM assumes exponential behavior both at the beginning and at the end of the curve, this model requires the adjustment of only the asymmetry exponent $\alpha$ (in addition to the parameters $r$ and $K$ ), being therefore the most robust model regarding convergence. In general, the RM is more suitable for epidemic curves in an intermediate stage, but it may also apply to curves in more advanced stages for which neither the BLM nor the GRM are suitable.

Once the theoretical model (among the BLM, GRM and $\mathrm{RM})$ that best describes the data is determined, the Modlnterv computes the characteristic points $t_{i}$ and thus determines the stage of the epidemic in that locality, according to the classification scheme described above.

If, however, the ModInterv identifies an empirical curve in the stage of increasing acceleration, the fitting of the data is redone with the $q$-exponential model given in (11). This is necessary because during the early growth regime the empirical curve is still far from developing an inflection point, so that the theoretical prediction for $t_{c}$ obtained from the RM or the GRM will lie well beyond the last date $t_{f}$ and hence this estimate for $t_{c}$ is not reliable. More to the point, in such case there are not enough data yet to yield a reliable estimate of the parameter $\alpha$, and hence it is preferable to use the $q$ exponential model, which describes only the initial growth. As discussed before, the $q$-exponential allows us to make projections into the near future, such as the doubling time $T_{d}$; see (13). The value of $T_{d}$ is automatically computed by the ModInterv algorithm when it identifies that the epidemic data in question is better described by the $q$-exponential model, and the value of $T_{d}$ is presented in the corresponding legend box, together with the values of $q$ and $r$. 
Once the model that best describes a given empirical curve has been identified, the Modlnterv app displays the figures of the cumulative and daily curves, showing the empirical data together with the respective theoretical fits. The figure title identifies the location in question and informs the model that best adjusted the curve, while the legend box gives the fitting parameters as well as the epidemic stage of that location. In the corresponding figure of the cumulative curve for the selected location, the app also shows, by means of vertical lines of appropriate colors, all the characteristic times $t_{i}, i=$ $2, \ldots, 5$, up to the current date, namely those for which $t_{i}<$ $t_{f}$. These temporal marks represent estimates as to when in the past the respective empirical curve progressed from one epidemic stage to the next. The last of such vertical in the cumulative curve thus helps to make visually clear what is the current state of the epidemic in that location.

\subsection{Numerical Analysis}

All numerical data fittings implemented in the Modlnterv are performed via Levenberg-Marquardt (LM) algorithm to solve the corresponding nonlinear optimization problem by the least squares method. More specifically, for the $q$ exponential model the LM routine fits formula (11), so as to determine the parameters $r$ and $q$. Similarly, for the Richards model the routine tries to fit the curve given in (8) to the selected data, thus obtaining the best numerical estimates for the parameters $(r, \alpha, K)$.

To fit the beta logistic model and the generalized Richards model, a slightly different approach is adopted. Given that the exact solution for these models is written in implicit form, as shown in (2), the LM routine treats the empirical data in the same implicit form, namely as a set of points $\left(C_{n}, t_{n}\right)$. In the case of the BLM, we then fit equation (8), viewed as a function $t(C)$, to these points, so as to determine the five parameters $(r, q, \alpha, p, K)$. Similar procedure is applied to the GRM, since it corresponds to the particular case $p=1$ of the BLM, in which case only four parameters $(r, q, \alpha, K)$ need to be determined.

The computational codes to perform the respective fits are written in the Python language and employ the LM algorithm implemented in the lmfit package for Python, which has a built-in routine for estimating the errors of the fitted parameters via the covariance matrix (Newville et al. 2014). In the Modlnterv, the results of the fitting procedure are deemed acceptable when the errors in the parameters are smaller than a certain threshold. For the analysis presented here, the error tolerance was set at $20 \%$ of the values estimated for the parameters themselves.
Stages of COVID-19 in the Brazilian States

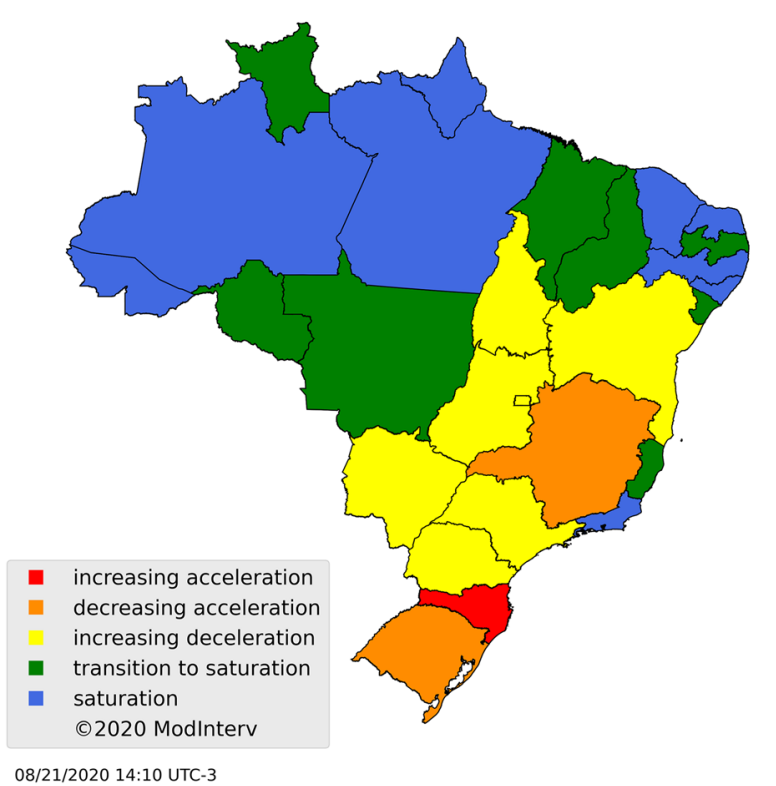

Fig. 2 Situation of the COVID-19 epidemic in the Brazilian States and Federal District, on August 21, 2020, according to the classification of the epidemic stages implemented in the ModInterv application

\section{Results}

In Fig. 2, we show the map of Brazil, in which each state and the Federal District are painted with the color corresponding to the respective stages of the COVID-19 epidemic on August 21, 2020. As already mentioned, this date was chosen because it corresponds to a period in which all five stages of the COVID-19 epidemic are represented in Brazil. The map shows that distribution of the epidemic in a continental country like Brazil is very heterogeneous. Below we discuss this situation in more detail.

In Figs. 3, 4, 5, 6, 7, 8, 9 and 10, we present the plots produced by the ModInterv for each of the Brazilian federal units, organized into groups according to the respective stages of the epidemic. In the left panel of each figure, we present the empirical data (red circles) and the theoretical curves (black curve) for the cumulative number of deaths attributed to COVID-19 for the respective federal unit.

In Figs. 3, 4, 5, 6, 7, 8, 9 and 10, the right panels show the corresponding daily number of deaths, together with the derivative $\mathrm{d} C / \mathrm{d} t$ of the theoretical curve $C(t)$ shown in the respective left panel. Below we discuss in more detail the results for each of the respective groups.

\subsection{State in the Stage of Increasing Acceleration}

The empirical curve for the state of Santa Catarina is best adjusted by the $q$-exponential growth model, as shown in the 


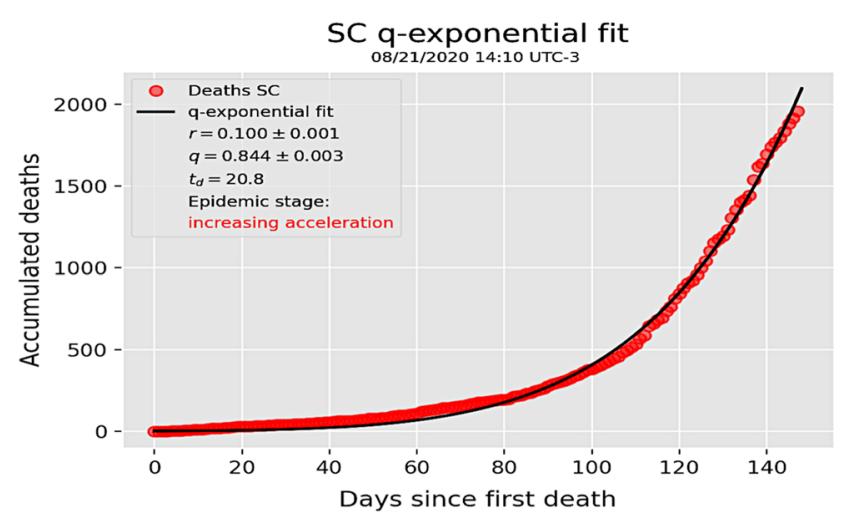

Fig. 3 Left panel: cumulative number of deaths attributed to COVID19 in the Brazilian state of Santa Catarina (SC), up to August 21, 2020. The red circles are the empirical data, and the solid black curve indicates the fit by the $q$-exponential model. Right panel: empirical daily number

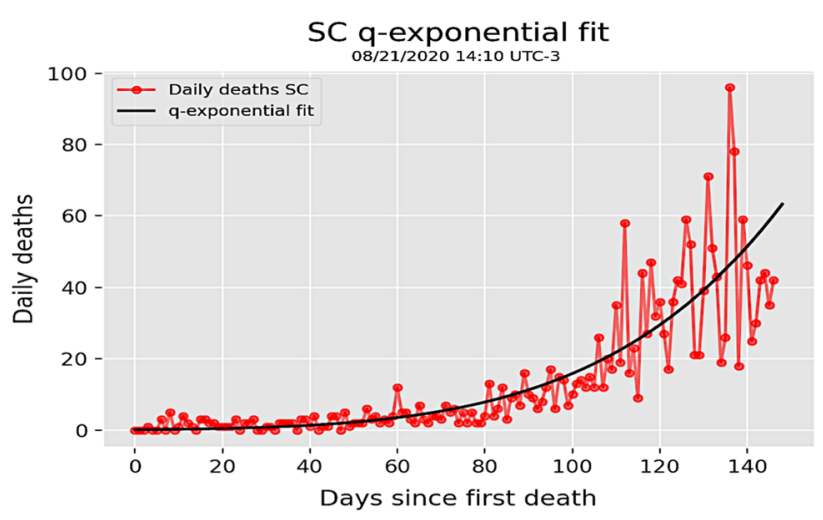

of deaths (red circles) and the theoretical prediction (solid black curve) corresponding to the time derivative of the solid curve in the left panel. Santa Catarina was the only state in the stage of increasing acceleration up to the time considered here
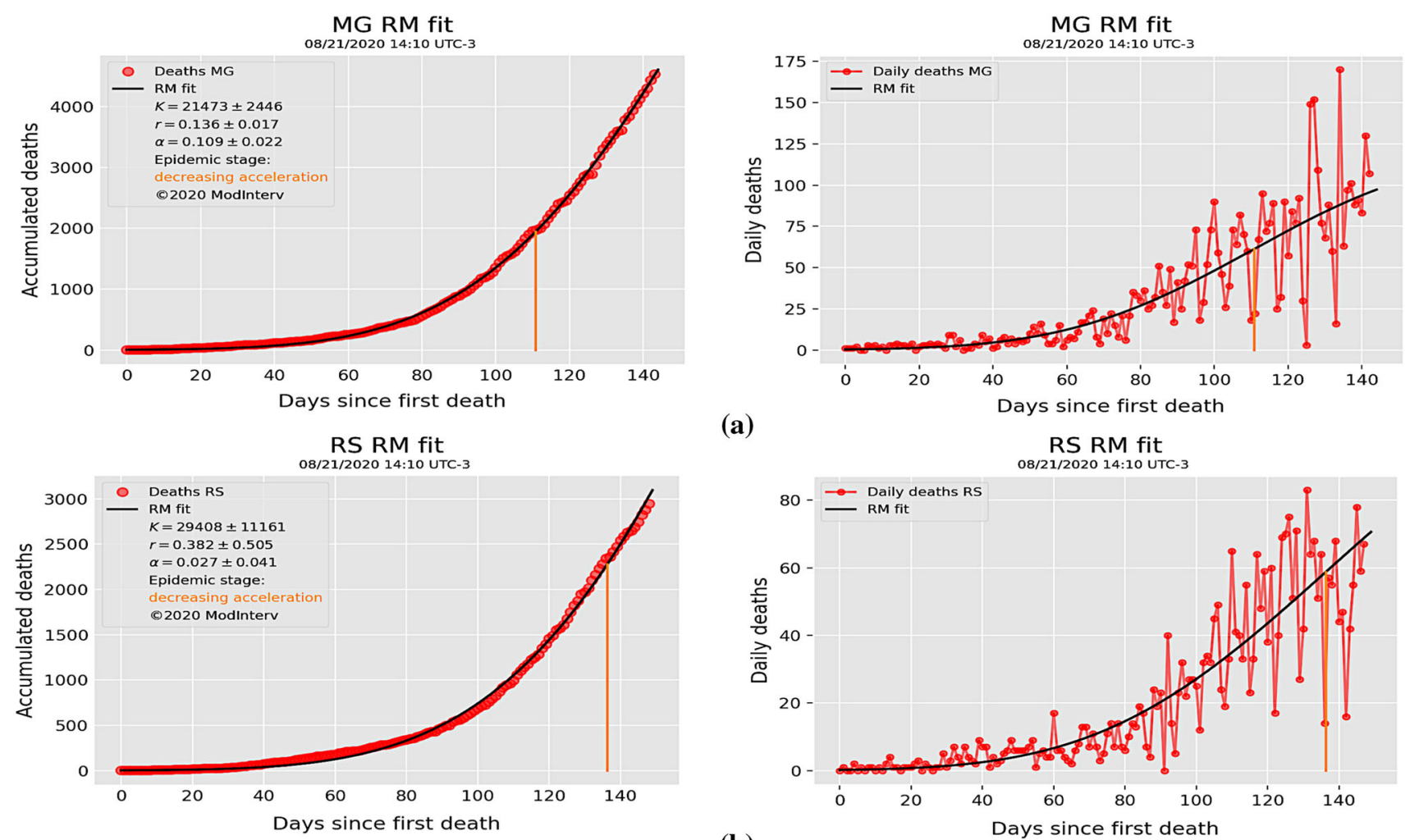

(a)

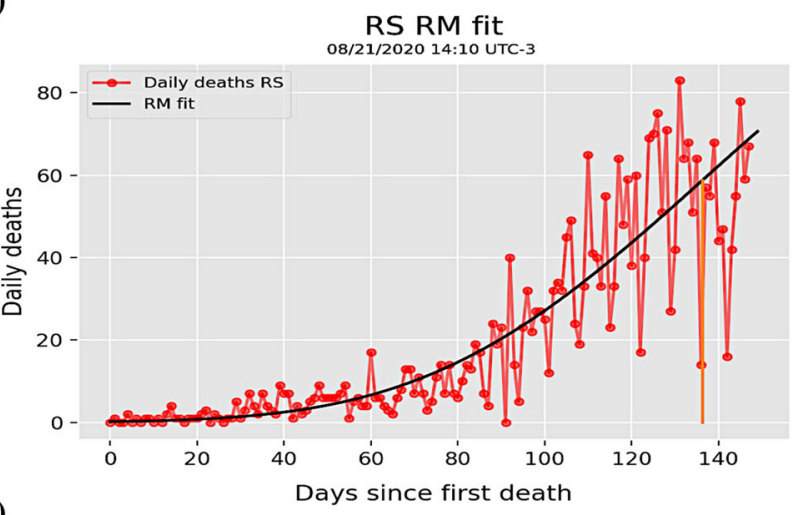

(b)

Fig. 4 Same as in Fig. 3 for the states of Minas Gerais (MG) in (a) and Rio Grande do Sul (RS) in (b). Here the black lines represent fits by the Richards model (RM). The orange vertical lines denote the location of the point $t_{2}$, indicating that the two states shown here are in the stage of decreasing acceleration

left panel of Fig. 3, indicating that in this state the COVID-19 fatality curve was still in a dynamical regime of increasing acceleration. In the right panel of Fig. 3, we show the daily number of deaths, together with the derivative of the theoretical curve, where we can see that the agreement between data and theory is also very good for the daily data.
From Fig. 3, we observe a linear growth, which goes approximately up to 60th day after the first death.

This shows that in the first two months the epidemic curve for Santa Catarina exhibited a regime of slow growth (i.e., with near zero acceleration, i.e., $q \approx 0$ ), as a reflection of the non-pharmacological intervention measures adopted at 

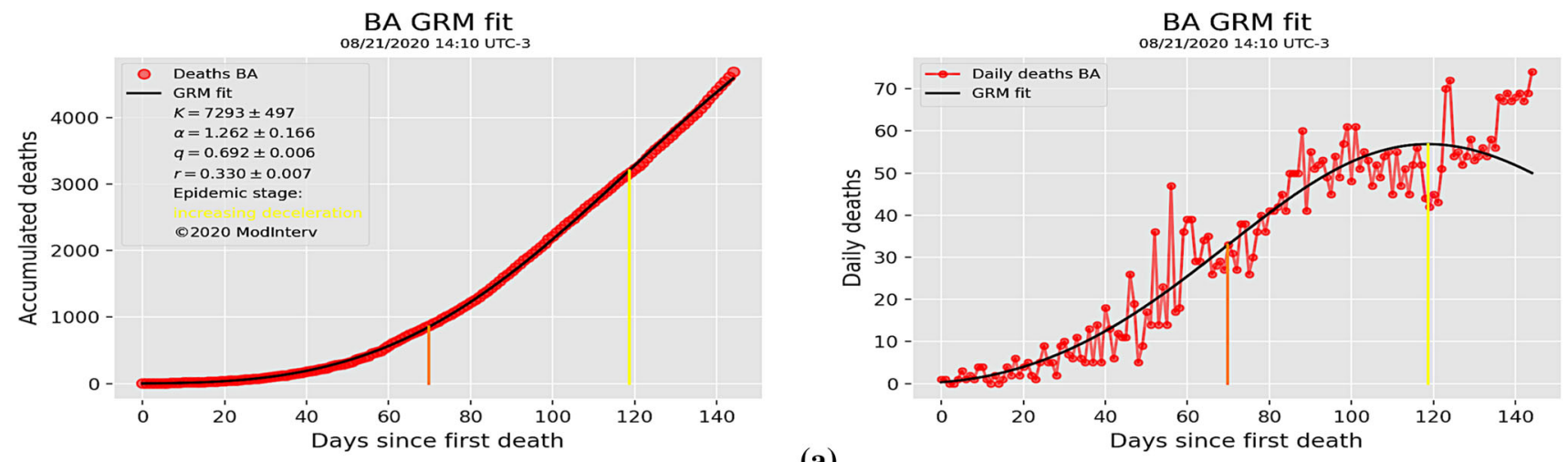

(a)

DF RM fit

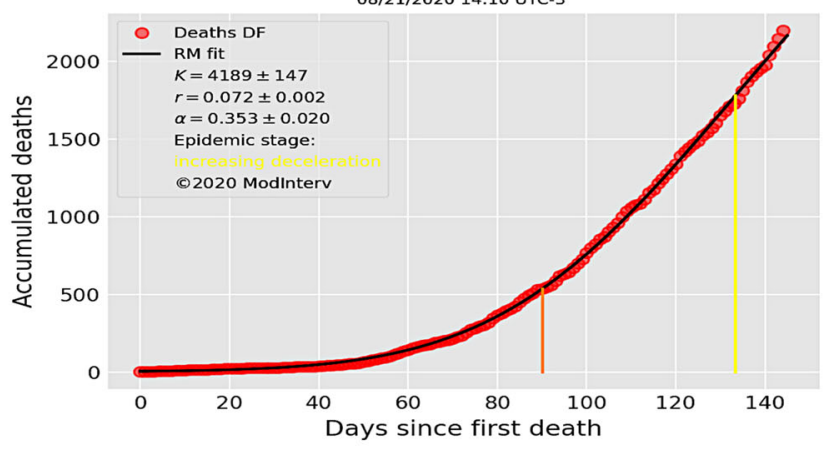

GO RM fit

(b)

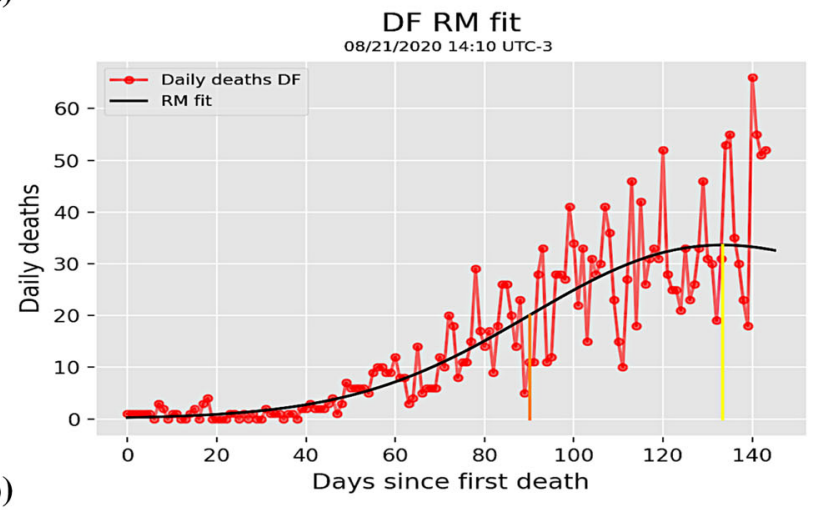

08/21/2020 14:10 UTC-3
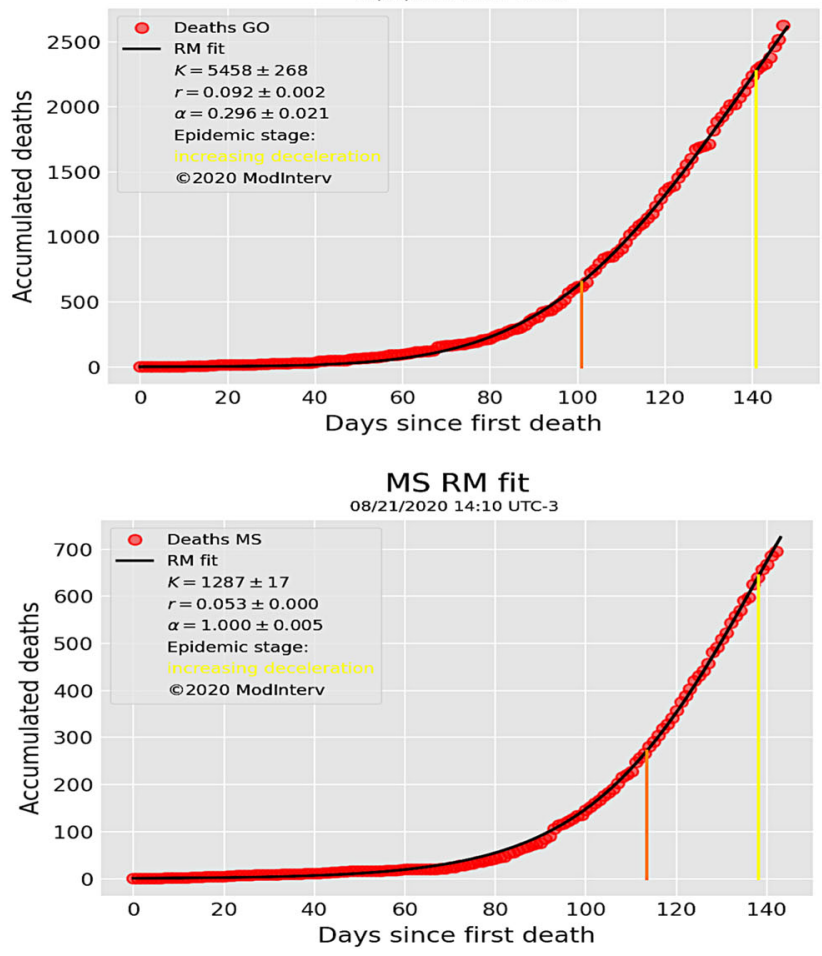

(c)
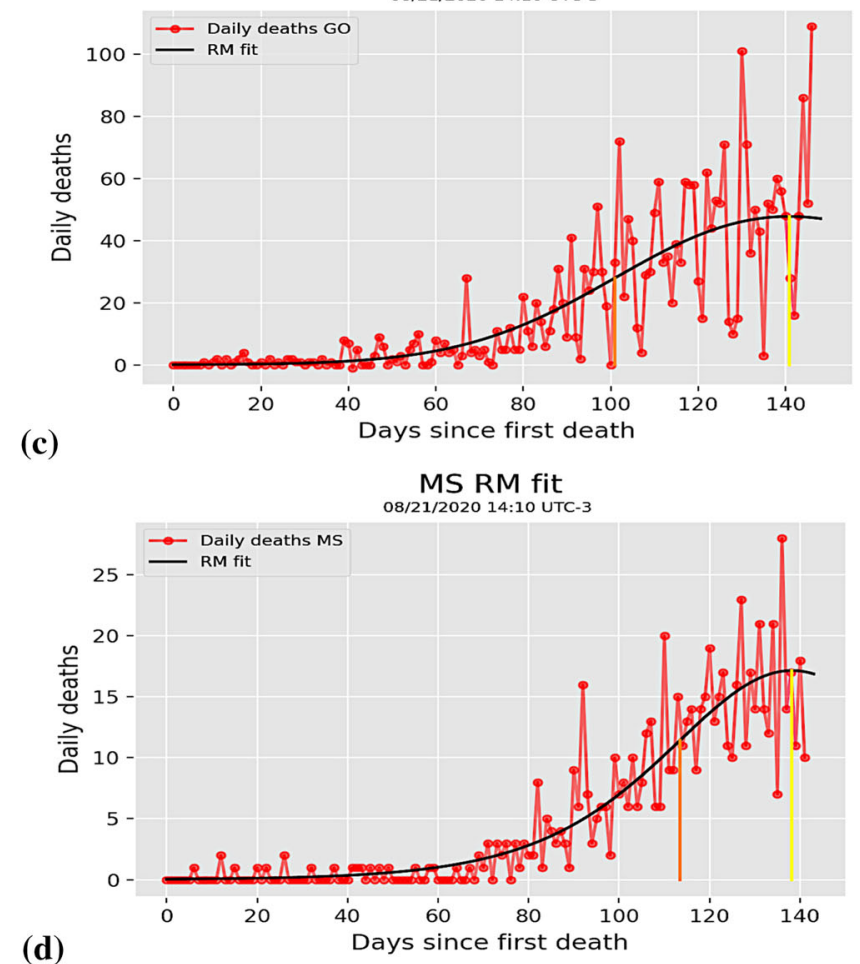

Fig. 5 Same as in Fig. 3 for the states of Bahia (BA), Distrito Federal (DF), Goiás (GO), and Mato Grosso do Sul (MS), from (a-d), respectively. Here, the black lines represent fits by either the generalized Richards model (GRM) or the Richards model (RM). The orange

and yellow vertical lines denote the location of the points $t_{2}$ and $t_{3}$, respectively, indicating that the states shown here are in the stage of increasing deceleration 

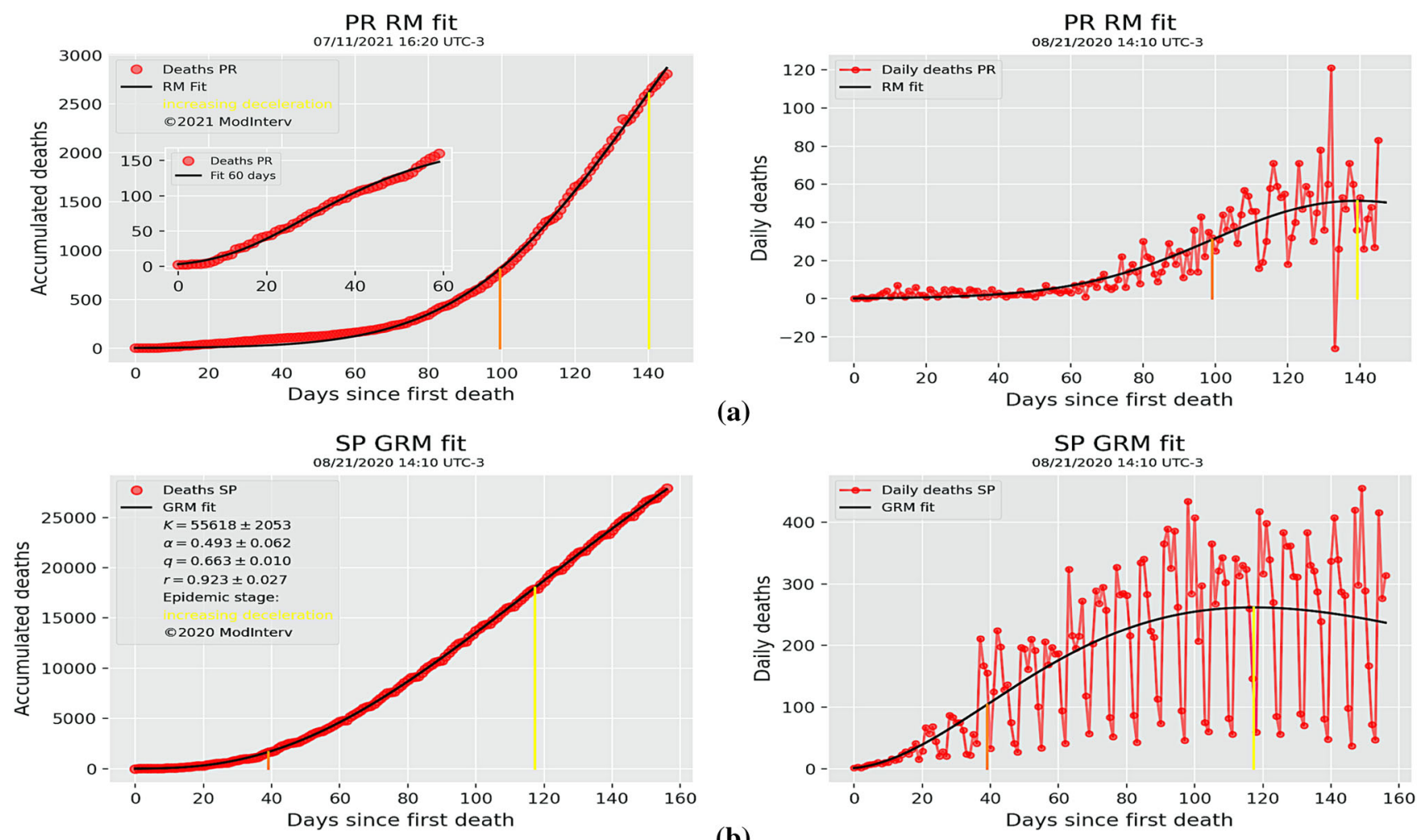

(a)
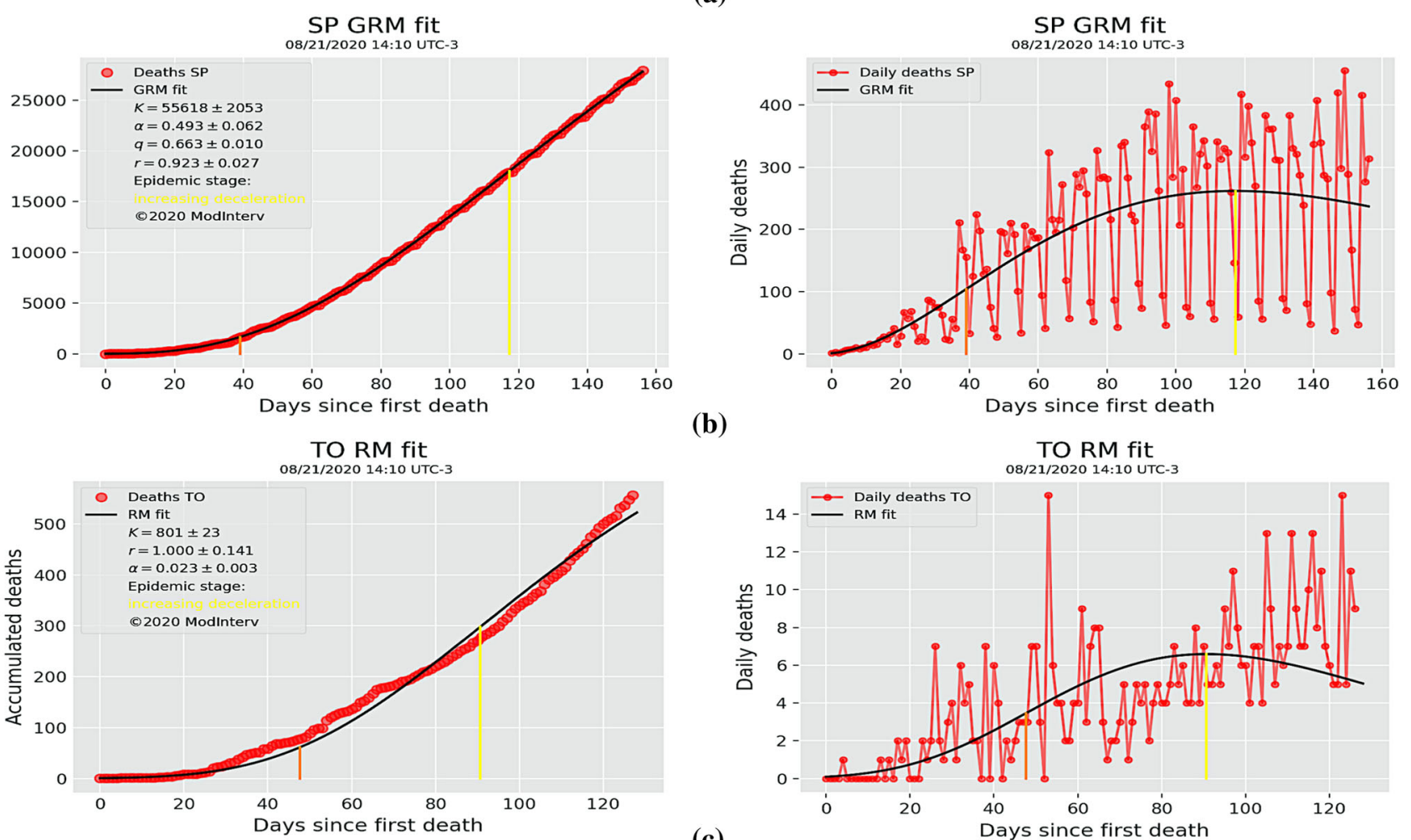

(b)

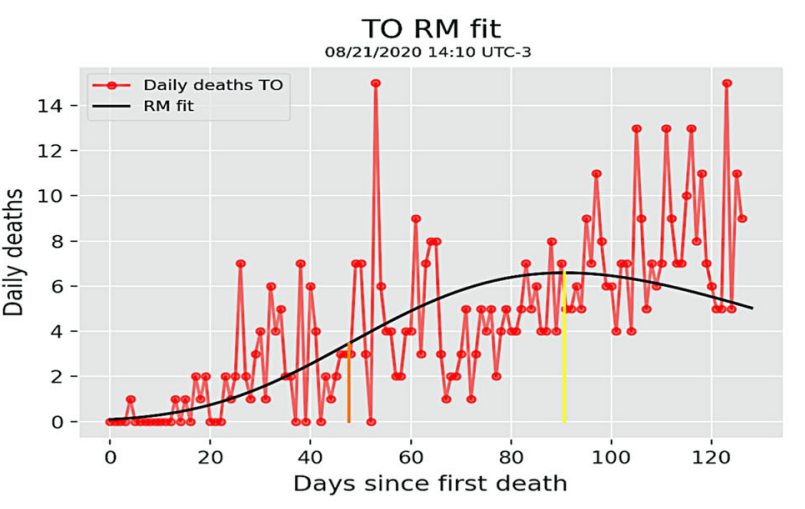

Fig. 6 Same as in Fig. 5 for the states of Paraná (PR), São Paulo (SP), and Tocantins (TO), from (a-c), respectively, which are also in the stage of increasing deceleration

the beginning of the pandemic, such as closure of schools, universities, and non-essential businesses.

Later, with the premature relaxation of these measures, notably the reopening of some economic activities, the curve quickly changed trajectory, passing to exhibit an almost exponential growth (i.e., with $q$ close to 1 ).

\subsection{States in the Stage of Increasing Deceleration}

As seen in Figs. 5 and 6, the states of Bahia, Goiás, Mato do Grosso do Sul, Paraná, São Paulo, Tocantins, and Distrito Federal are in the second stage of the intermediate phase, which is characterized by an increasing deceleration.

\subsection{States in the Stage of Decreasing Acceleration}

The states of Minas Gerais and Rio Grande do Sul are in the first stage of the intermediate growth regime, namely the stage of decreasing acceleration, as shown in Fig. 4. It can be seen from this figure that the last point of each of the empirical curves had already exceeded the orange line, corresponding to the point $t_{2}$ of maximum acceleration, but there had not yet appeared the formation of the inflection point in the empirical curve, thus characterizing the second epidemic stage, as defined before.

One can also see from Fig. 4 that the epidemic curves for the states of Minas Gerais and Rio Grande do Sul exhibit a sudden change in trend around the second month of the epi- 

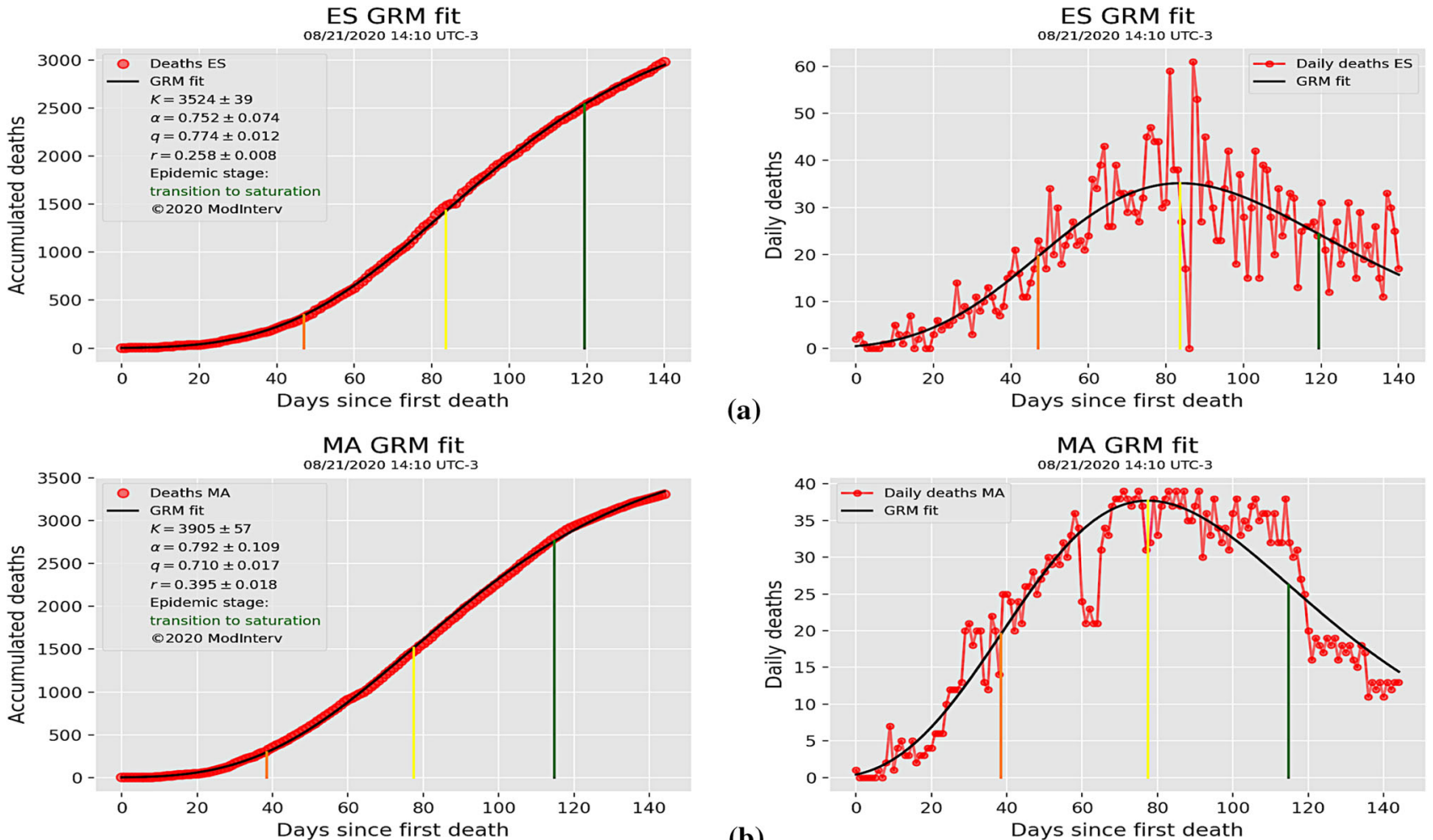

(a)
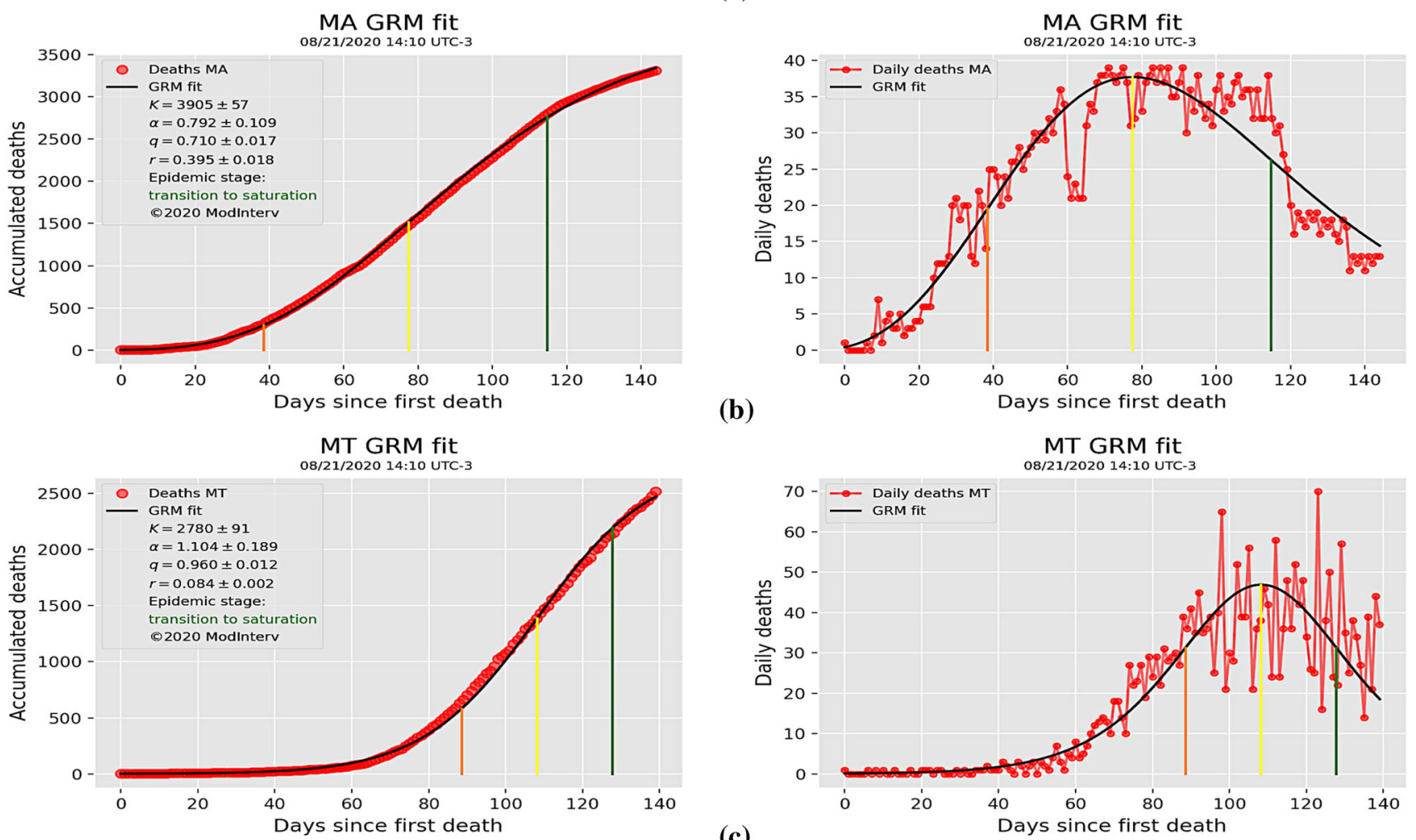

PB GRM fit

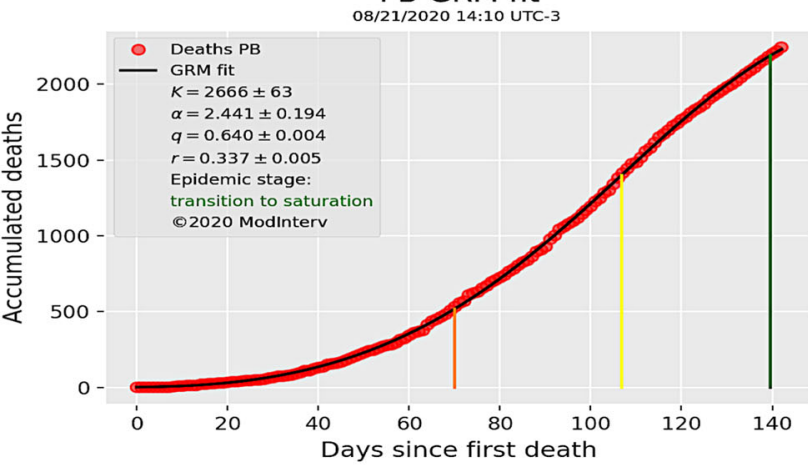

(c)

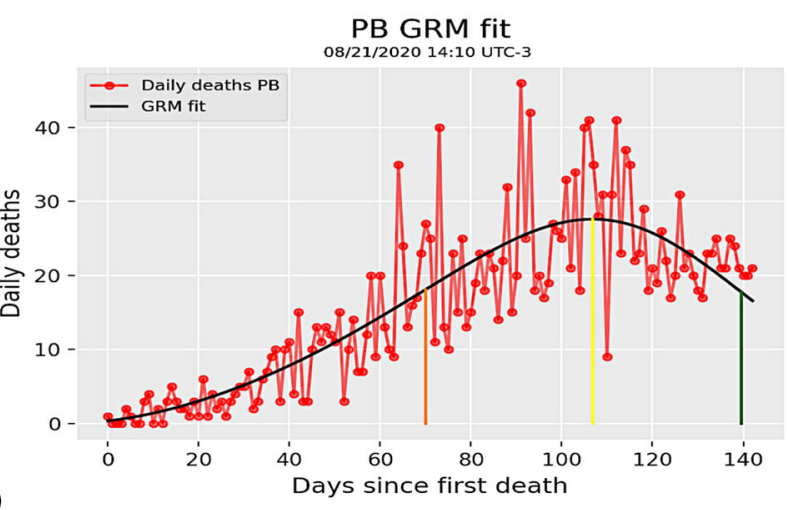

(d)

Fig. 7 Same as in Fig. 3 for the states of Espírito Santo (ES), Maranhão (MA), Mato Grosso (MT), and Paraíba (PB), from (a-d), respectively. Here, the black lines represent fits by the generalized Richards model (GRM) 


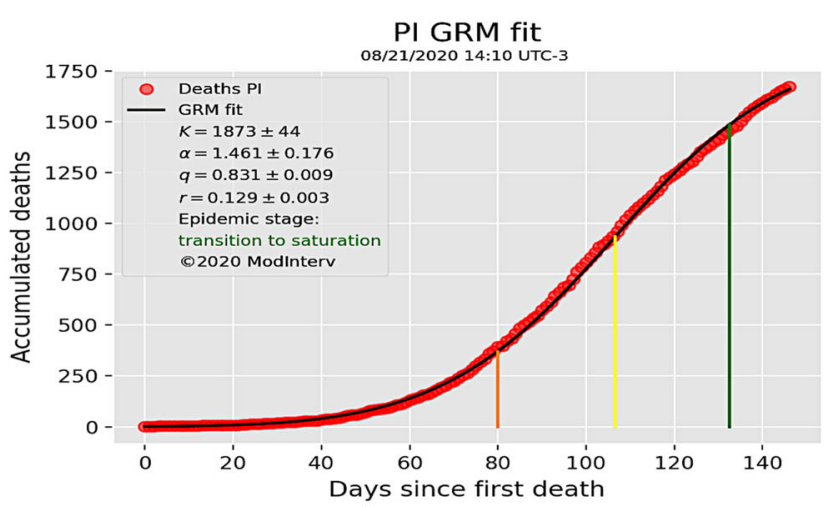

(a)

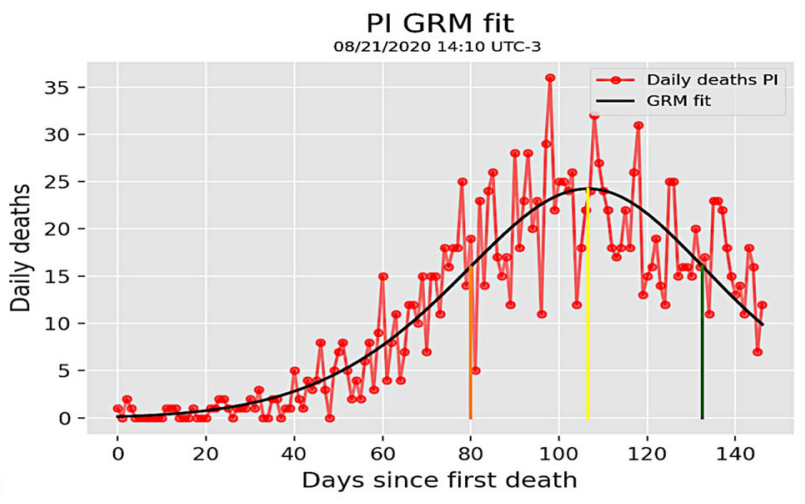

RO RM fit

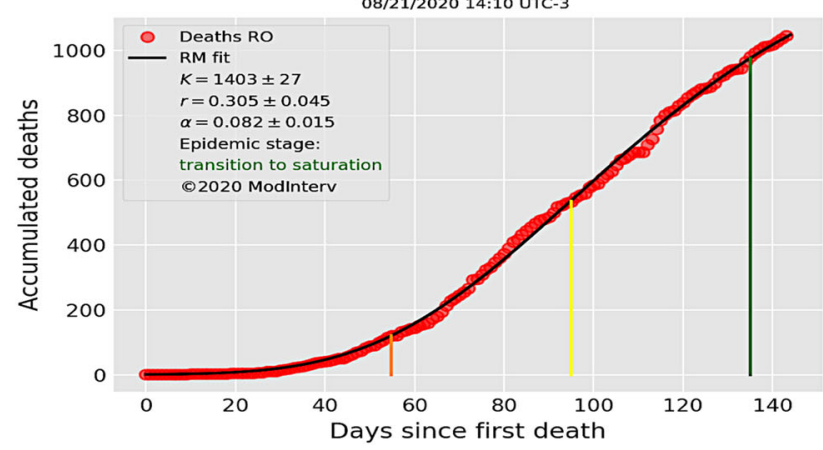

(b)
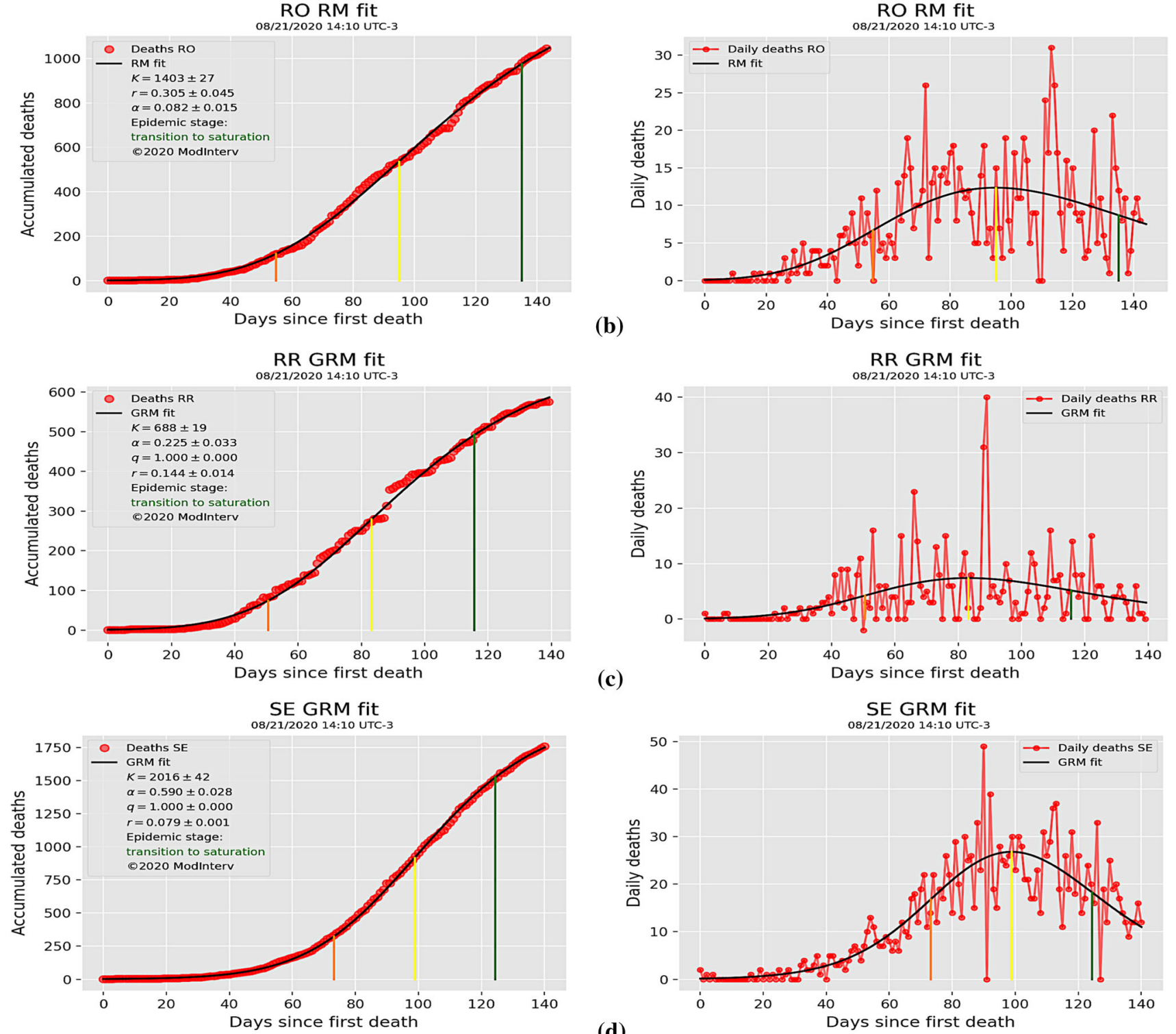

(c)

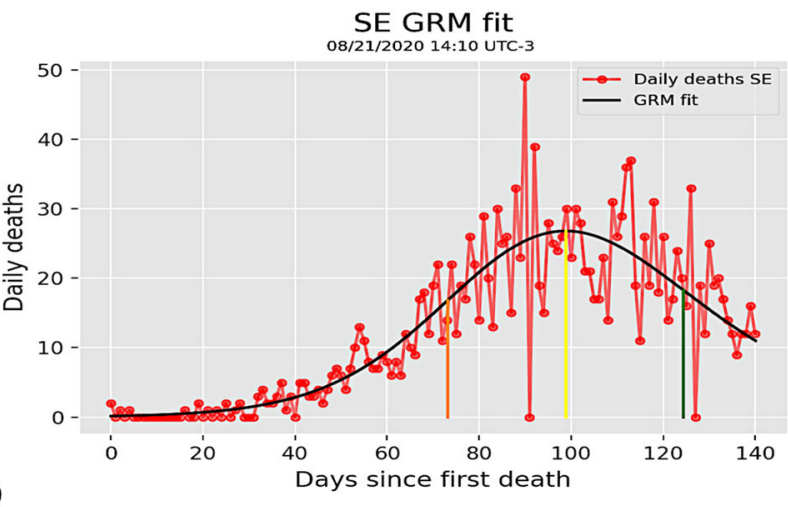

Fig. 8 Same as in Fig. 7 for the states of Piauí (PI), Rondônia (RO), Roraima (RR), and Sergipe (SE), from (a-d), respectively, which are also in the transition to saturation stage 

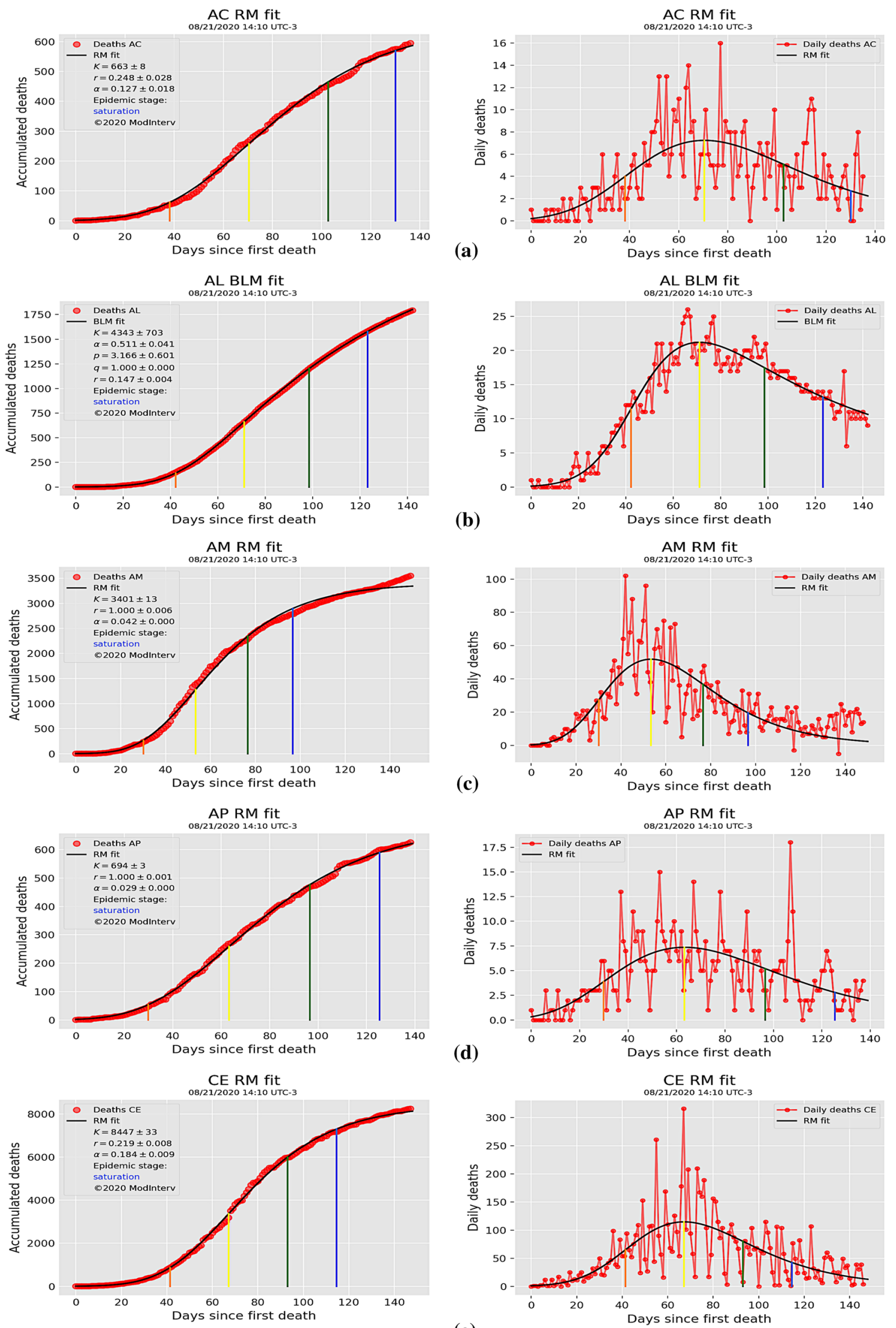

(e)

Fig. 9 Same as in Fig. 3 for the states of Acre (AC), Alagoas (AL), Amazonas (AM), Amapá (AP), and Ceará (CE), from (a-d), respectively. Here, the black lines represent fits by either the logistic beta model (BLM) or the Richards model (RM) 

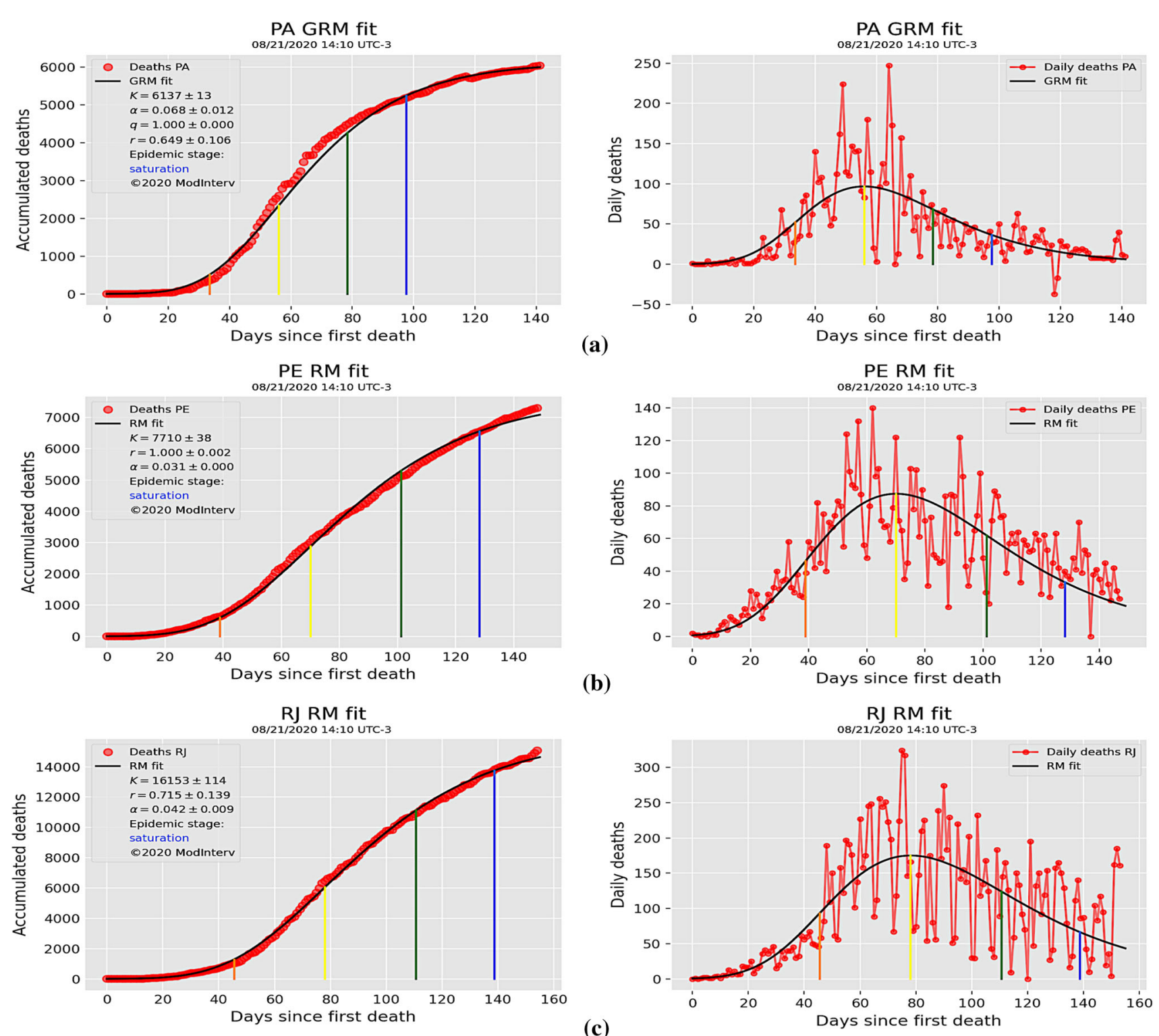

(b)
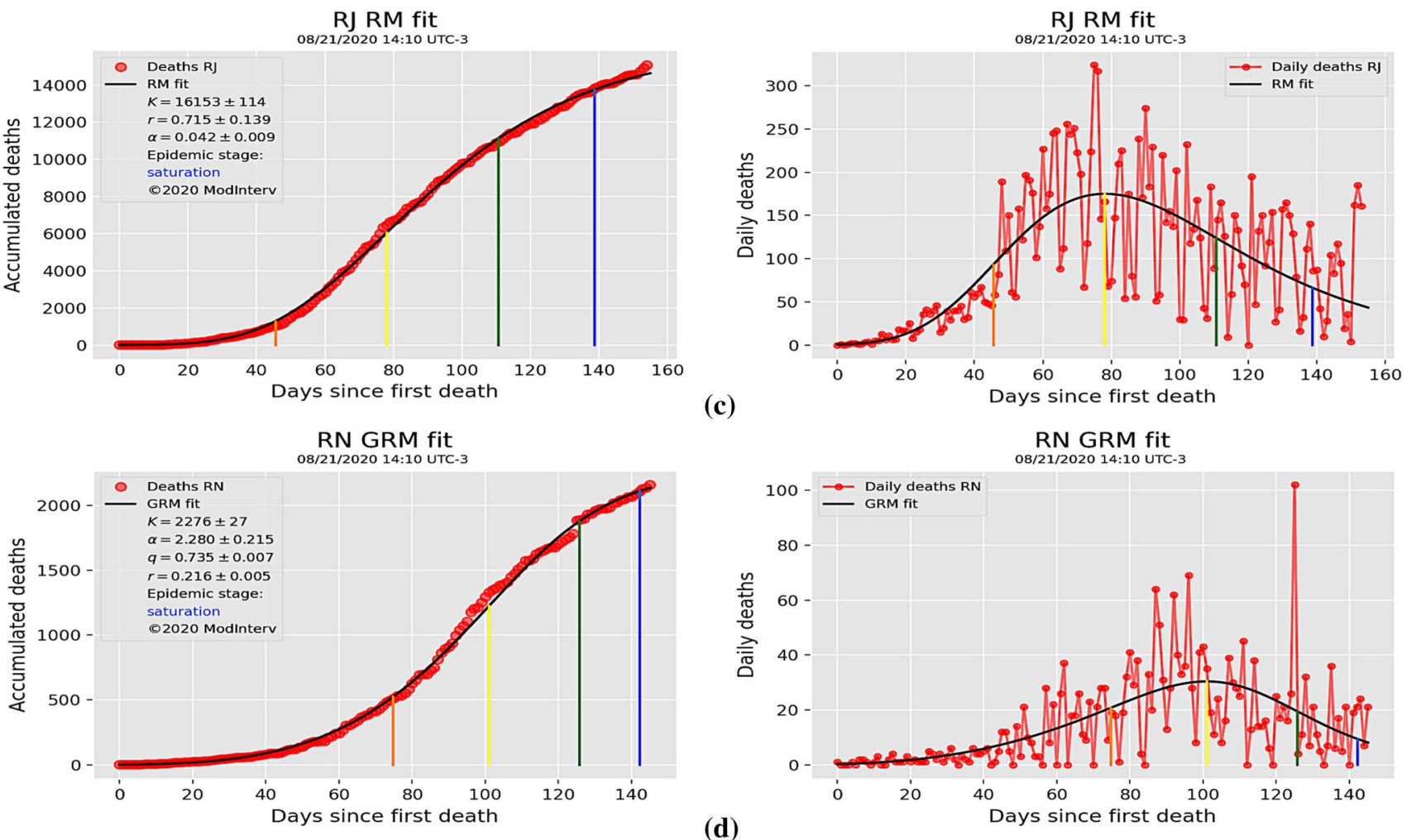

Fig. 10 Same as in Fig. 9 for the states of Pará (PA), Pernambuco (PE), Rio de Janeiro (RJ), and Rio Grande do Norte (RN), from (a-d), respectively, which are also in the saturation stage 
demic, passing from a linear regime to a quasi-exponential growth. This quick change in trend is similar to what happened to the epidemic curve of Santa Catarina around the same time (see discussion above) and is probably due to the same reasons (i.e., premature relaxation of control measures). However, at the moment of the present analysis, the states of Minas Gerais and Rio Grande do Sul had already left the regime of increasing acceleration (where Santa Catarina still was) and progressed to the stage of decreasing acceleration.

The mathematical fits suggest that the epidemic curves for these states had already passed through the inflection point (indicated by the yellow line), and so they had started the deceleration phase of the epidemic, but they had not yet reached the point $t_{4}$ of maximum deceleration, hence the epidemic curves had not entered the late growth regime.

From Figs. 5 and 6, one can also estimate the times when the respective curves progressed from one epidemic stage to the next, as indicated by the orange and yellow vertical lines. In particular, one sees that the state of Paraná exhibits a nearly linear growth regime for almost the first two months, as was also observed for the states shown in Figs. 3 and 4. This slow growth is more visible in the inset of the left panel in Fig. 6a, where we show the empirical data and the respective fit by the RM only up to day 60 . In the inset one sees that there was a slight deceleration after the first month, so that a projection of this trend (if the epidemiological conditions remained the same) would imply that the epidemic would eventually progress toward a saturation, as suggested by the solid line in the inset which is beginning to bend. This slow early growth in the state of Paraná can be attributed, as discussed before, to the early adoption of non-pharmacological measures. However, shortly before day 60 one sees a strong accelerated growth which was caused by the relaxation of the control measures.

When seen in the larger time scale of the main plot of the left panel in Fig. 6a, the evolution up to day 100 (vertical orange line) can be said to belong to the overall increasing acceleration stage, given that the first wave in the state of Paraná only started to be brought effectively under control after day 140, as indicated by the main inflection point (yellow vertical line).

\subsection{States in the Stage of Transition to Saturation}

The states of Espírito Santo, Maranhão, Mato Grosso, Paraíba, Piauí, Rondônia, Roraima, and Sergipe were found to be in the stage of transition to saturation, as shown in Figs. 7 and 8 . As previously discussed, this stage is the first stage of the late growth regime and is characterized by the fact that the epidemic curve has already passed the point $t_{4}$ of maximum deceleration (green line). Therefore, it is already in a regime of decreasing deceleration but still with an increasing jerk. This growing jerk forces the curve to deviate away from the near-linear growth of the intermediate phase, thus contributing to the initial bending of the epidemic curve. We can thus say, with a certain degree of reliability, that for the states at this stage the epidemic (or rather its first wave) was beginning to get under control.

\subsection{States in the Saturation Stage}

Finally, we have the states of Acre, Alagoas, Amazonas, Amapá, Ceará, Pará, Pernambuco, Rio de Janeiro, and Rio Grande do Norte, which are already in the stage of saturation properly, as can be seen in Figs. 9 and 10. At this stage, the epidemic curve has already surpassed the blue line, which corresponds to the point $t_{5}$ of maximum jerk. Therefore, at this last stage of the epidemic, the velocity, acceleration, and jerk of the epidemic curve are all decreasing functions of time, so that the epidemic is approaching its final plateau. Therefore, it may be said that at this stage the epidemic is relatively under control (assuming, of course, that the saturation trend is maintained over time).

\section{Discussion}

In this work, we have applied four mathematical growth models, implemented in the ModInterv application, to study the COVID-19 fatality curves of the Brazilian States and the Federal District, up until August 21, 2020. Up to that date, nearly five months after the first death in Brazil, all five epidemic stages were represented in at least one state throughout the country.

We verified, in particular, that Santa Catarina was the only state in the early growth regime, where the epidemic curve was still exhibiting an increasing acceleration. The other two states of the Southern region, Rio Grande do Sul and Paraná, had recently emerged from this early growth regime and were already in the first and second stages of the intermediate growth regime, corresponding to the stages of decreasing acceleration and increasing deceleration, respectively. Thus, all three Southern states were at that time far from curtailing the epidemic.

In contradistinction, all states in the Northern and Northeastern regions, except for Bahia and Tocantins, were either in stage of transition to saturation (Maranhão, Paraíba, Piauí, Rondônia, Roraima, and Sergipe) or in the saturation stage (Alagoas, Amazonas, Amapá, Ceará, Pernambuco, and Rio Grande do Norte), which are the two final stages of the epidemic (if there is no recrudescence of infections). Bahia and Tocantins were in the stage of increasing deceleration, but had not yet reached the transition to the saturation stage.

Among the Midwestern states, only Mato Grosso had already started the transition to saturation, while Goiás, Mato Grosso do Sul and the Federal District were still in the stage 
of increasing deceleration. The Southeastern region had the highest diversity of epidemic stages, in the sense that each state was in a different stage, namely: Minas Gerais was in the stage of decreasing acceleration, São Paulo was in the stage of increasing deceleration, Espírito Santo was in the stage of transition to saturation, and Rio de Janeiro was the only Southeastern state which was already in the saturation stage.

The present analysis allows the interesting conclusion that five months onto the COVID-19 epidemic in Brazil the Northern and Northeastern regions were, in general, in a relatively more advanced phase of control of the pandemic, whereas in the more Southern states the epidemic was still accelerating or displayed only a slight deceleration. This general overview of the COVID-19 situation in Brazil largely reflects the distinct evolution patterns of the epidemic in the different regions of the country. For example, in many capitals of the Northern and Northeastern states, there was a strong accelerated growth in the number of deaths in the first few months of the epidemic, which forced local authorities to adopt more drastic measures to contain the spread of the virus.

These measures contributed to the subsequent "bending" of the epidemic curve that we have seen in our analysis for these states. In contrast, in the Southern states, the evolution of the disease happened somewhat in the reverse: the initial growth of the epidemic curve was slow because of the early mitigation measures; but later on these measures were prematurely relaxed, which led, in turn, to a resurgence of the epidemic.

As a consequence of this "relapse" of the epidemic, most Southern states were still in a phase of acceleration or, at most, of mild deceleration at the time of the present analysis. Other states that presented this "relapse effect" were Goiás, Mato Grosso do Sul and Minas Gerais, meaning that these states also had a slow early growth in the first couple of months (nearly with a constant acceleration, i.e., $q \approx 0$ ) but then experienced a quick surge in the number of COVID-19 deaths. As a result, by mid-August, 2020, they were far from the saturation phase, with Minas Gerais still in the decreasing acceleration phase and Goiás and Mato Grosso do Sul in the increasing deceleration phase. Another interesting example is the state of São Paulo, which maintained mitigation measures for a considerable period of time. These measures caused the curve to leave the stage of increasing acceleration relatively early, see Fig. 6b, but were not sufficient to "bend" the curve and interrupt the almost linear behavior of the intermediate growth regime, so that the state still remained in the stage of increasing deceleration.

As we have argued in Introduction, knowledge of the current stage of an epidemic in a given locality might provide useful information to the local health and government authorities. For example, in the case of COVID-19 it was often the case that strict non-pharmacological interventions, such as closure of schools and non-essential businesses, were implemented in the early stages of the epidemic (Maier and Brockmann 2020; Vasconcelos et al. 2020); but deciding when to start relaxing them was a difficult decision for health and political authorities (Vasconcelos et al. 2021b). Ideally, interventions should be relaxed only after the daily numbers of new cases or deaths have passed their peaks, i.e., when the epidemic enters a deceleration phase. It is therefore important to detect when the epidemic growth curve reaches a regime of decreasing acceleration, as it signals that the peak of the daily curve (or the inflection point of the cumulative curve) is near, so that authorities should start planning for the relaxation of control measures. Such relaxation, however, should be implemented only after it becomes evident that the epidemic curve has entered the regime of increasing deceleration. A practical use along theses lines of the Modlnterv app (together with other epidemiological analyses) was made by the COVID-19 data analysis team of the Prefecture of Teresina, capital of Piauístate (Soares-Filho and Câncio 2021).

Detailed knowledge of the dynamical stages of an epidemic in its final phase is also relevant for health authorities. For example, once the epidemic enters a regime of decreasing deceleration, meaning that the worst phase has (supposedly) been left behind, it becomes important to monitor the velocity of this deceleration (jerk), while the jerk is still increasing (stage 4) or if it only recently started to decrease (beginning of stage 5), it might be premature to further relax the control measures, as there is a considerable risk of a resurgence of the epidemic, causing a second wave of infections, as discussed in more detail by Vasconcelos et al. (2021a). Further to this point, we note that although the effective reproduction number $R_{t}$ can identify the peak of the daily curves (at which point it reaches unity), it cannot by itself provide sufficient information about the different acceleration regimes. Hence it can be quite useful to have a fuller description of the epidemic evolution, as we have shown in the present study.

In a large country such as Brazil, it is important for Federal and State public health authorities to be aware of possible geographical patterns concerning the evolution of an epidemic. We have seen above that states of the same geographical region tend to display similar evolution patterns, albeit not necessarily synchronized in time. This was particularly evident, for example, in the states of the Southern and Northeastern regions, respectively. This shows that the health authorities of a given state should also pay attention to what is happening in neighboring states, as they can learn from their neighbors' experiences and thus take measures in advance either to prevent repetition of an undesirable pattern (say, strong accelerated growth) or to achieve a similar outcome (say, control of the epidemic), depending on the specific situation. For instance, Curitiba, the capital of Paraná state, was among the first capitals in Brazil to relax control mea- 
sures in mid-April 2020 (Sfair et al. 2020), when the fatality curve was still accelerating (albeit slowly). This later led to an explosion of cases, which in turn forced the city authorities to reimpose control measures in June 2020 (Justi 2020). A similar path-i.e., relaxation of mitigation measures before the epidemic was decelerating - was adopted by the other Southern capitals, with similarly disastrous results, as reflected in the "relapse effect" mentioned above for the Southern states. Had the authorities from, say, Rio Grande do Sul state, followed more closely what was happening in Paraná State, perhaps they would have chosen a different course of action.

The results of the present study, which identified similarities but also considerable differences in the geographical evolution of COVID-19 in Brazil, are further evidence that a central coordination of the efforts in the fight against the pandemic is greatly desirable, so as to avoid as much as possible a heterogeneous pattern among the states and regions, which presumably makes it more more difficult to bring the epidemic under control within the entire country.

It should be emphasized that the mathematical analysis presented here revealed the evolution of the COVID-19 epidemic in Brazil up to the maximum date (August 21, 2020) considered in our analysis. The trajectories of the epidemic in the Brazilian federal units after that date have been strongly influenced by the maintenance or relaxation of control measures in the respective states. An analysis of the "full history" of the COVID-19 epidemic in Brazil, up to the present time, is beyond the scope of this paper. But a few comments are in order. For example, by October 9, 2020, all states had progressed to the late growth regime, i.e., they were either in the transition to saturation or in the saturation stages, indicating that the "first wave" of the epidemic was by then getting under control in Brazil. However, in late October and early November, 2020, the epidemic curves of several states started to show an upsurge, thus deviating from the up-to-then saturation trend.

Such a recrudescence of the epidemic was likely the consequence of the so-called "second wave" of infections (Vasconcelos et al. 2021a). These effects will be studied later, since they require the use of more complex models with timedependent parameters.

Another interesting application of the epidemic stage classification scheme introduced here would be to analyze the evolution of COVID-19 within individual states in Brazil. Many Brazilian states are, for administrative purposes, further divided into Health Districts ("Regionais de Saúde" in Portuguese), as this decentralized organization helps to better carry out the state's sanitary and public health policies. One could then apply our model to each health district of a given state, so as to determine the stages of the epidemic in all the districts at a desired moment in time. This would contribute to providing a better view of the evolution and progression of the epidemic in that state. However, as the epidemic data are not readily available for the states' health districts, our algorithm would need first to aggregate the data by health districts, which would require additional work on the ModInterv app. This type of analysis will be particularly relevant in the event that we implement a corresponding classification scheme for multiple-wave epidemics, as suggested above, since most (if not all) states in Brazil have thus far displayed at least two waves of COVID-19. This is therefore an interesting avenue of research to explore in the future.

Acknowledgements An early version of this paper was presented at the XXIII Congresso Brasileiro de Automática (CBA 2020). This work was partially supported by the National Council for Scientific and Technological Development $(\mathrm{CNPq})$ in Brazil, through the grants Nos. 303772/2017-4 (GLV), 312612/2019-2 (AMSM), and 305305/2019-0 (RO). AAB thanks CNPq for its support through a PhD Fellowship (Grant No. 167348/2018-3). GLV also acknowledges partial funding from UFPR through the COVID-19/PROIND-2020 Research Program. RO thanks D.A.D.O. and CASTLab. The authors thank the editor and anonymous referees for comments and suggestions.

\section{Declarations}

Conflict of interest The authors declare that they have no known competing financial interests or personal relationships that could have appeared to influence the work reported in this paper.

Ethical Approval No animal or human studies were carried out by the authors in this work; hence, ethical permission is not required.

\section{References}

Adam, D. (2020). A guide to R-The pandemic's misunderstood metric. Nature, 583, 346-348.

Almagor, J., \& Picascia, S. (2020). Exploring the effectiveness of a COVID-19 contact tracing app using an agent-based model. Scientific Reports, 10(1), 1-11.

Anderson, R., Donnelly, C., Hollingsworth, D., Keeling, M., Vegvari, C., Baggaley, R., \& Maddren, R. (2020). Reproduction number $(R)$ and growth rate $(r)$ of the COVID-19 epidemic in the UK: Methods of estimation, data sources, causes of heterogeneity, and use as a guide in policy formulation. The Royal Society 2020. https://royalsociety.org/-/media/policy/projects/set-c/ set-covid-19-R-estimates.pdf.

Baunez, C., Degoulet, M., Luchini, S., Pintus, P. A., \& Teschl, M. (2021). Tracking the dynamics and allocating tests for COVID-19 in real-time: An acceleration index with an application to French Age Groups and Départements. PLOS ONE, 16(6), 1-21. https:// doi.org/10.1371/journal.pone.0252443.

Brum, A. A., Duarte-Filho, G. C., \& Vasconcelos, G. L. (2020). Application ModInterv COVID-19. http://fisica.ufpr.br/modinterv.

Bürger, R., Chowell, G., \& Lara-Díaz, L. Y. (2019). Comparative analysis of phenomenological growth models applied to epidemic outbreaks. Mathematical Biosciences and Engineering: MBE, 16(5), 4250-4273.

Chowell, G. (2017). Fitting dynamic models to epidemic outbreaks with quantified uncertainty: A primer for parameter uncertainty, identifiability, and forecasts. Infectious Disease Modelling, 2(3), 379-398. 
Cota, W. (2020). Monitoring the number of COVID-19 cases and deaths in Brazil at municipal and federative units level. https://covid19br. wcota.me/.

El-Morshedy, M., Altun, E., \& Eliwa, M. (2021). A new statistical approach to model the counts of novel coronavirus cases. Mathematical Sciences. https://doi.org/10.1007/s40096-021-00390-9.

Johns Hopkins University. (2021). Coronavirus COVID-19 Global Cases by the Center for Systems Science and Engineering (CSSE) at Johns Hopkins University (JHU). https://coronavirus.jhu.edu/ map.html. Accessed: January 30, 2021.

Justi, A. (2020). Após relaxamento, prefeitura anuncia novas medidas de combate ao coronavírus em Curitiba. Portal G1 PR shorturl.at/jAI35.

Keeling, M. J., \& Rohani, P. (2011). Modeling infectious diseases in humans and animals. Princeton University Press.

Kermack, W., \& McKendrick, A. (1927). A contribution to the mathematical theory of epidemics. Proceedings of the Royal Society of London Series A, Containing Papers of a Mathematical and Physical Character, 115(772), 700-721.

Macêdo, A. M., Brum, A. A., Duarte-Filho, G. C., Almeida, F. A., Ospina, R., \& Vasconcelos, G. L. (2021). A comparative analysis between a SIRD compartmental model and the Richards growth model. Trends in Computational and Applied Mathematics, 22(4), 545-557.

Maier, B. F., \& Brockmann, D. (2020). Effective containment explains subexponential growth in recent confirmed COVID-19 cases in China. Science, 368(6492), 742-746.

Manchein, C., Brugnago, E. L., da Silva, R. M., Mendes, C. F., \& Beims, M. W. (2020). Strong correlations between power-law growth of COVID-19 in four continents and the inefficiency of soft quarantine strategies. Chaos: An Interdisciplinary Journal of Nonlinear Science, 30(4), 041102.

Newville, M., Stensitzki, T., Allen, D. B., \& Ingargiola, A. (2014). LMFIT: Non-linear least-square minimization and curve-fitting for Python, Chicago, IL. https://doi.org/10.5281/zenodo.11813.

Picoli, S., Jr., Mendes, R., Malacarne, L., \& Santos, R. (2009). qdistributions in complex systems: A brief review. Brazilian Journal of Physics, 39(2A), 468-474.

Read, J. M., Bridgen, J. R., Cummings, D. A., Ho, A., \& Jewell, C. P. (2021). Novel coronavirus 2019-nCoV (COVID-19): Early estimation of epidemiological parameters and epidemic size estimates. Philosophical Transactions of the Royal Society B, 376(1829), 20200265.

Rebhan, E. (2021). Novel mathematical approach to COVID-19 studies with reproduction-like number and acceleration parameter. Research Square. https://doi.org/10.21203/rs.3.rs-303322/v1.

Sfair, A. (2020). Curitiba vai orientar reabertura do comércio de rua e uso de máscara será obrigatório. ParanáPortal, https://paranaportal.uol.com.br/cidades/curitiba-reaberturacomercio-de-rua.
Shamil, M. S., Farheen, F., Ibtehaz, N., Khan, I. M., \& Rahman, M. S. (2021). An agent-based modeling of COVID-19: Validation, analysis, and recommendations. Cognitive Computation. https:// doi.org/10.1007/s12559-020-09801-w.

Soares-Filho, F. S., \& Câncio Jr, G. S. (2021). Private communication.

Tsoularis, A., \& Wallace, J. (2002). Analysis of logistic growth modelsD. Math Biosciences, 179(1), 21-55.

Utsunomiya, Y. T., Utsunomiya, A. T. H., Torrecilha, R. B. P., Paulan, S. C., Milanesi, M., \& Garcia, J. F. (2020). Growth rate and acceleration analysis of the COVID-19 pandemic reveals the effect of public health measures in real time. Frontiers in Medicine, 7, 247. https://doi.org/10.3389/fmed.2020.00247.

Vasconcelos, G. L., Brum, A. A., Almeida, F. A. G., Macêdo, A. M. S., Duarte-Filho, G. C., \& Ospina, R. (2021a). Standard and anomalous waves of COVID-19: A multiple-wave growth model for epidemics. Brazilian Journal of Physics, 51, 1867-1883. https:// doi.org/10.1007/s13538-021-00996-3.

Vasconcelos, G. L., Macêdo, A. M., Duarte-Filho, G. C., Brum, A. A., Ospina, R., \& Almeida, F. A. (2021b). Power law behaviour in the saturation regime of fatality curves of the COVID-19 pandemic. Scientific Reports, 11, 4619. https://doi.org/10.1038/s41598-02184165-1.

Vasconcelos, G. L., Macêdo, A. M., Ospina, R., Almeida, F. A., DuarteFilho, G. C., Brum, A. A., \& Souza, I. L. (2020). Modelling fatality curves of COVID-19 and the effectiveness of intervention strategies. PeerJ, 8, e9421. https://doi.org/10.7717/peerj.9421.

Wang, X. S., Wu, J., \& Yang, Y. (2012). Richards model revisited: Validation by and application to infection dynamics. Journal of Theoretical Biology, 313, 12-19.

Worldometer, (2020). Worldometer-COVID-19 data. https://www. worldometers.info/coronavirus/. Accessed: June 30, 2021.

Wu, K., Darcet, D., Wang, Q., \& Sornette, D. (2020). Generalized logistic growth modeling of the COVID-19 outbreak: Comparing the dynamics in the 29 provinces in China and in the rest of the world. Nonlinear Dynamics, 101(3), 1561-1581.

Publisher's Note Springer Nature remains neutral with regard to jurisdictional claims in published maps and institutional affiliations. 